\title{
Vortex dynamics of a jet at the pressure node in a standing wave
}

\author{
Nicholas A. Worth ${ }^{1} \uparrow$, Dhiren Mistry ${ }^{1}$, Tim Berk ${ }^{2}$ and James R. Dawson ${ }^{1}$ \\ ${ }^{1}$ Department of Energy and Process Engineering, Norwegian University of Science and Technology, \\ 7491 Trondheim, Norway \\ ${ }^{2}$ Department of Aerospace Engineering and Mechanics, University of Minnesota, Minneapolis, \\ MN 55455, USA
}

(Received 25 April 2019; revised 22 August 2019; accepted 6 October 2019)

In this paper we investigate the vortex structure and dynamics formed in the near field of a turbulent axisymmetric jet subjected to transverse acoustic forcing. Full three-dimensional phase-averaged velocity measurements were obtained to elucidate the coherent structures formed when the jet is positioned at the pressure node of a plane standing wave oriented transversely to the streamwise flow direction, which creates a plane symmetry about the nodal line dissecting the jet exit. Due to the change in phase that occurs across the nodal line, it was found that axisymmetry is broken and the jet undergoes a periodic transverse flapping motion consistent with a sinuous mode. This was accompanied by a periodic train of interconnected vortex structures, resembling inverted hairpin (or horseshoe) vortices, formed as the shear layers rolled up in anti-phase either side of the jet, and propagated a few diameters downstream before breaking up. An inviscid vortex model employing inverted hairpin line vortices is shown to capture both the dynamics of the vortex structures and the fluctuating velocity fields. Overall, the jet response and resulting vortex dynamics observed represent a significant departure from the axisymmetric flow structures observed with conventional longitudinal forcing and more closely resemble the phenomenon of bifurcating jets.

Key words: jets, vortex dynamics

\section{Introduction}

It has long been known that the Kelvin-Helmholtz instability leads to the formation of organised coherent structures in the near field of turbulent jets (Becker \& Massaro 1968; Crow \& Champagne 1971). Depending on their application, there has been significant interest in methods to manipulate the Kelvin-Helmholtz instability mechanism by taking control of the jet boundary conditions through some form of excitation in order to control the formation of coherent structures, to understand 
the fundamental response of jets to perturbations or to influence various aspects of the downstream flow development such as the evolution of turbulent statistics, mixing rates and amplification or suppression of jet noise amongst others (Crow \& Champagne 1971; Hussain \& Zaman 1980; Zaman \& Hussain 1980; Gutmark \& Ho 1983; Ho \& Huerre 1984; Zaman 1985; Reynolds et al. 2003; Sadeghi \& Pollard 2012). In the vast majority of cases these effects are considered in response to axisymmetric disturbances of finite amplitude.

There have been comparatively few studies to understand the response of jets to asymmetric forcing and in particular transverse forcing. Reynolds et al. (2003) summarised an early set of experimental studies in which jets were subjected to asymmetry using a combination of axial and helical forcing. This caused the momentum field of the jet to split (or bifurcate) into two or more directions, and in some cases rapid spreading through the unstable so-called blooming phenomenon. To produce the asymmetric disturbances, complex forcing methods were used such as mechanically oscillating the jet nozzle (Lee \& Reynolds 1985) and acoustically by phased oscillations directed around the nozzle circumference (Parekh, Leonard \& Reynolds 1988). This induced either a slight staggering, or inclination change in successive ring structures which, through mutual induction, proceed to alter their relative orientation and phase as they advect downstream, effectively amplifying the asymmetry, with dramatic consequences for the far-field jet development. This reorientation of structures through mutual induction was demonstrated through a series of vortex filament simulations, which are described in Leonard (1985). Similar dynamics has also been produced by employing alternative methods of active forcing (Suzuki, Kasagi \& Suzuki 2004; Kasagi 2006) or passive control (Longmire \& Duong 1996).

More recently numerical simulations have also provided further insight into the phenomenon of jet bifurcation. These have demonstrated that a dramatic enhancement of the jet spreading rate could be achieved solely through transverse forcing rather than through a combination of axial and transverse forcing (Urbin \& Metais 1996; Danaila \& Boersma 2000). They have also examined the dependence of spreading rates on Strouhal number (da Silva \& Métais 2002), as well as the interaction between axial and flapping modes of excitation in determining the symmetry of the far-field response (Tyliszczak \& Geurts 2014) with the ratio of axial to helical excitation frequencies being able to control the jet blooming phenomena by dividing the momentum stream into more than two branches (Gohil, Saha \& Muralidhar 2015; Tyliszczak 2015). While the primary focus of these investigations is often stated as the modification or control of the far-field jet behaviour in terms of spreading rates, some studies have also begun to qualitatively describe the organisation of coherent structures in the near field. For example, da Silva \& Métais (2002) observed an arrangement of inclined vortex rings formed during flapping excitation, with the alternate tilting and pairing of subsequent vortices resulting in a large merged train of coherent vortices. A similar, complex near-field reorganisation of coherent structures was observed by Urbin \& Metais (1996), Danaila \& Boersma (2000) and Suzuki et al. (2004).

The current study is motivated by the important practical problem of self-excited thermoacoustic instabilities in annular combustor geometries which are relevant to gas turbines and rocket engines (Wolf et al. 2012; Worth \& Dawson 2013; Bauerheim et al. 2014; Bourgouin et al. 2015; Ghirardo, Juniper \& Moeck 2016). If we consider a rotationally symmetric annular geometry with a number of equispaced jet-stabilised flames around the circumference, thermoacoustic instabilities typically 
excite the azimuthal acoustic modes of the annulus. This generic case corresponds to jet-stabilised flames immersed in an approximately one-dimensional azimuthal acoustic field where the base flow of each jet is subjected to time-varying pressure gradients that are orthogonal (locally transverse) to the streamwise flow direction. Improved understanding of the effect of asymmetric forcing on the formation of coherent structures is important in turbulent premixed flames as they directly affect the fluctuating heat release rate through changes in the flame surface area (Trouvé \& Poinsot 1994).

Recently, the response of swirling flames (Hauser, Lorenz \& Sattelmayer 2011; O'Connor \& Lieuwen 2011, 2012) and laminar flames (Lespinasse, Baillot \& Boushaki 2013) to transverse excitation has been investigated using forcing configurations specifically designed to be long in the transverse direction, relative to the streamwise flow direction, to generate plane standing waves. The flame response, and by inference the flow response as premixed flames are stabilised on the shear layers, have shown significant departures from axisymmetric behaviour such as variations in the size of the coherent structures formed on opposite sides of the flame (Lespinasse et al. 2013), and the presence of helical (Hauser et al. 2011; O'Connor \& Lieuwen 2011) and plane-symmetric flame dynamics (Dawson \& Worth 2014, 2015). However, the more fundamental case of the response of a canonical round jet immersed in a transverse acoustic field has yet to be investigated despite being a fundamental building block to more complex engineering applications such as thermoacoustic instabilities in annular combustor geometries.

In this paper we investigate the vortex structure and dynamics formed in the near field of a turbulent axisymmetric jet positioned at the pressure node of a plane standing wave generated by transverse acoustic forcing. This represents an as yet unexplored method of applying asymmetric excitation in comparison with previous studies of bifurcating jets. In contrast to the above mentioned studies of bifurcating jets which primarily focus on the far field, we concentrate on the near-field response which is relevant to the problem of thermoacoustic instabilities in annular combustion chambers. In $\S 2$ we describe the transverse forcing method and apparatus, how the phase-resolved volumetric particle image velocimetry (PIV) measurements were obtained to elucidate the three-dimensional coherent structures formed in the near field, and a vortex model. In $\S 3$ the vortex structure and dynamics are presented and analysed followed by the results of the vortex model. Finally, we summarise our findings in $\S 4$. In order to aid understanding of the vortex dynamics presented herein, two supplementary movies are available at https://doi.org/10.1017/jfm.2019.821.

\section{Experimental methods and modelling}

\subsection{Experimental set-up}

The axisymmetric jet, which was used in Aydemir, Worth \& Dawson (2012) and Lawson \& Dawson (2013), was placed at the base of a long rectangular box with a pair of loudspeakers mounted to adjustable end walls as illustrated in figure 1 . The walls were adjustable in order to change the resonance frequencies of the box and to vary the position of the jet relative to the acoustic mode. The speakers mounted at each end were used to produce approximately one-dimensional plane waves.

Air was supplied from a high-pressure supply via a mass flow controller (Alicat MCR-500) which ensured that the flow rate varied by less than $2 \%$ throughout the experiments. The flow was initially passed through a cylindrical plenum chamber with a length of $200 \mathrm{~mm}$ and diameter of $100 \mathrm{~mm}$ containing a honeycomb flow 
(a)

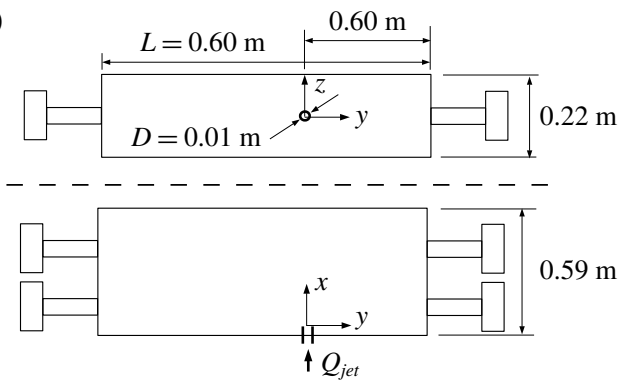

Schematic of enclosure

(c)

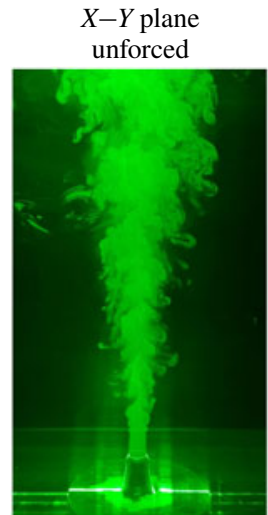

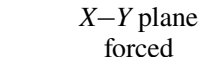

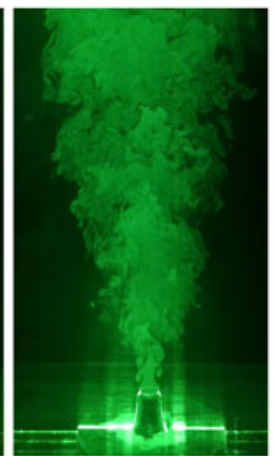

(b)

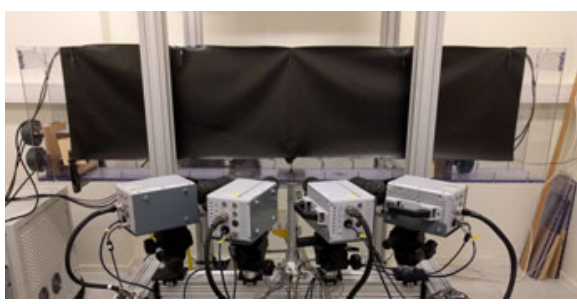

Image of enclosure and cameras

$$
\begin{array}{cc}
X-Z \text { plane } & X-Z \text { plane } \\
\text { unforced } & \text { forced }
\end{array}
$$

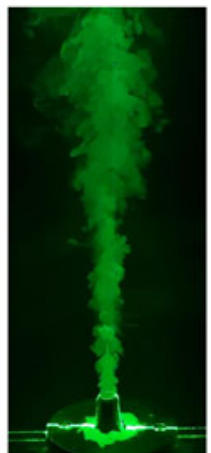

Visualisation of the jet comparing forced and unforced cases

FIgURE 1. (a) Schematic and (b) image of the enclosure, forcing and camera set-up, and coordinate system. (c) A Mie scattering visualisation of the forced and unforced jet in two cross-sectional planes, illustrating the modified spreading rates in the far field.

straightener, and then into a pipe with a length of $300 \mathrm{~mm}$ and diameter of $35 \mathrm{~mm}$, followed by a converging nozzle with an exit diameter of $D=0.01 \mathrm{~m}$. The jet exit was knife-edged, and the nozzle was designed with a cubic matched geometry to produce a top hat exit velocity profile. The exit nozzle protruded $30 \mathrm{~mm}$ into the box to avoid wall effects. A large box width to jet diameter ratio, $W / D=22$, was employed to ensure confinement effects were negligible in the near-field region of the jet, from $0 \leqslant x / D \leqslant 4$.

Standing waves were generated using four speakers (Monacor KU-516) positioned on opposing end walls. Resonance tubes were fitted to the end of each speaker, which were of length $\lambda / 4$, where $\lambda$ is the wavelength of the standing pressure wave. A signal generator (Aim-TTi TGA1244) connected to two $200 \mathrm{~W}$ amplifiers (Crown CE1000) was used to drive the speakers. This forcing signal was also used as a reference in order to phase-lock image acquisition. In order to characterise the acoustic mode the pressure in the rectangular box was measured at four transverse locations using microphones (Brüel \& Kjær 4939-A-011) mounted flush with the wall.

In the context of combustion instabilities, the Strouhal numbers of interest are selected based on approximate aero engine scaling where the circumference of the annular combustor is $O(1.5 \mathrm{~m})$, the burner diameter is $O(0.03 \mathrm{~m})$ and the bulk velocity is $O\left(30-60 \mathrm{~m} \mathrm{~s}^{-1}\right)$, which gives a typical range of Strouhal numbers $S t=f_{f} D / u_{e}=0.3-0.6$. Accordingly, the jet response was initially investigated using 
stereoscopic PIV for a range of forcing frequencies, $f_{f}$, and bulk flow velocities, $u_{e}$, resulting in Strouhal numbers in the range $S t=0.19-0.6$. During these tests, the forcing amplitude was also varied, with the amplitude of transverse velocity oscillations increased to up to approximately $20 \%$ of the bulk flow velocity. During self-excited instabilities, oscillations grow until they are limited by nonlinear effects as they reach limit cycle (Dowling 1997), and large oscillation amplitudes may be expected. Such amplitudes far exceed the small perturbations which cause the preferred mode to lock in to the forcing frequency. Therefore, although the Strouhal number range is close to the expected preferred Strouhal number of the jet (Ho \& Huerre 1984), the significant amplitude of excitation applied in the current study in general resulted in a significant jet response and characteristic vortex dynamics within the range $S t=0.34-0.5$. A single case was selected for the current investigation, which was chosen to be generally representative in terms of the dynamics of the wider dataset.

In order to accommodate limitations in laser power and ensure that the flow could be fully captured within the field of view, full three-dimensional velocity measurements were undertaken at a single Reynolds number of $R e=6000$, where $R e=$ $u_{e} D / v$, in which $u_{e}=9.0 \mathrm{~m} \mathrm{~s}^{-1}$ is the bulk flow velocity and $v=1.5 \times 10^{-5} \mathrm{~m}^{2} \mathrm{~s}^{-1}$ is the dynamic viscosity of air at room temperature. The jet was forced at $f_{f}=450 \mathrm{~Hz}$, corresponding to a Strouhal number of $S t=0.5$, at an amplitude of transverse oscillation of $10 \%$ of the bulk flow. This combination of parameters ensured that the resulting vortex dynamics remained within the field of view whilst also being generally representative of the wider dataset. To achieve resonance at this frequency, the box dimensions were adjusted to $L=1.60 \mathrm{~m}, H=0.59 \mathrm{~m}$ and $W=0.22 \mathrm{~m}$. The jet was positioned a distance of $0.60 \mathrm{~m}$ from one edge of the box, as shown in figure 1, at the location of the pressure node. Based on this frequency, it should be noted that in the near field the jet diameter is very small in comparison with the acoustic wavelength $(\lambda=0.8 \mathrm{~m})$, and therefore the jet is considered acoustically compact.

A typical power spectral density from a single microphone is shown in figure $2(b)$, illustrating that the frequency response of the box enclosure is dominated by the forcing frequency. The multiple microphone method (Jang \& Ih 1998) was used to estimate in a least squares sense the magnitude of incident, $p_{i}$, and reflected, $p_{r}$, plane waves in the enclosure, and balance the forcing signals sent to the speakers. Figure 2(c) shows a reconstruction of the pressure oscillation magnitude with varying position in the enclosure, and the amplitude of the pressure measurements at the four microphone locations, normalised by $p_{a}$, the atmospheric pressure. Using this approach a standing wave ratio of $\left(\left|p_{i}\right|-\left|p_{r}\right|\right) /\left(\left|p_{i}\right|+\left|p_{r}\right|\right)= \pm 0.01$ was maintained throughout the experiments. Figure $2(c)$ also shows that the normalised maximum amplitudes of the acoustic pressure oscillations are very small, and therefore the acoustics remain linear. The jet was located at $y / L=0$, which was the location of a pressure node and a velocity anti-node. Pressure measurements (not shown) also verified that the resonant modes of the box were decoupled from the upstream jet geometry. Figure $2(d)$ shows the variation of the transverse component of velocity over a forcing cycle. There is reasonable agreement between the acoustic particle velocity amplitude reconstructed from the multiple microphone method and the velocimetry measurements, which show that the amplitude of transverse velocity oscillations is around $10 \%$ of the bulk flow velocity, $u_{e}$.

The streamwise, transverse and cross-stream coordinates and velocities are denoted by $x, y$ and $z$, and $u, v$ and $w$, respectively. Figure 2(a) shows profiles of the 


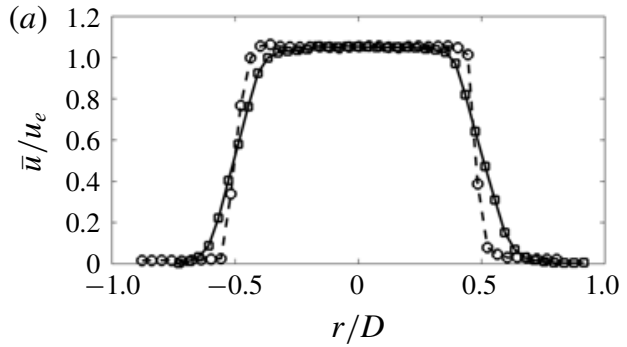

Mean streamwise velocity profiles

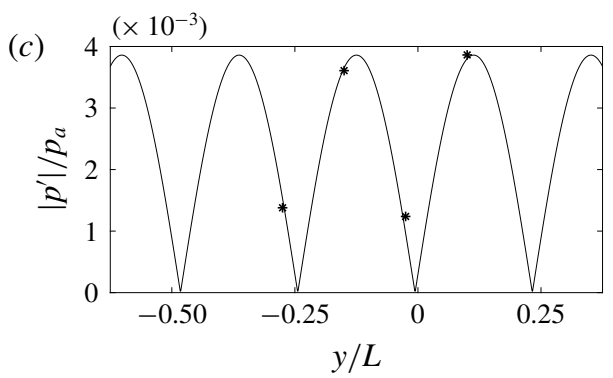

Magnitude of pressure oscillations in enclosure

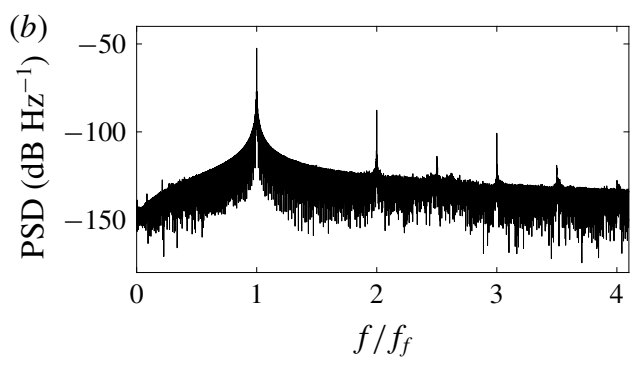

PSD from pressure measurement

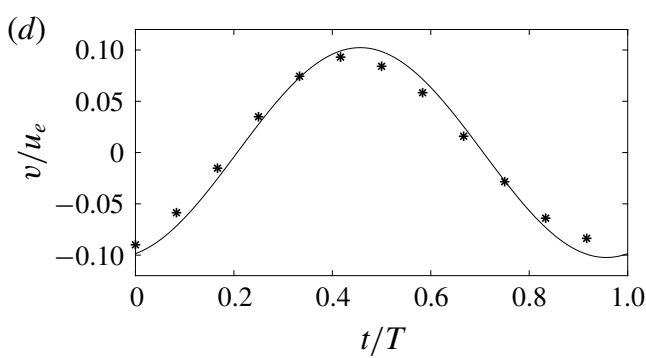

Transverse velocity oscillation

FIGURE 2. Jet and acoustic characterisation results. (a) Profiles of the mean streamwise velocity close to the jet exit. Profiles taken in the $x-y$ plane $(\square)$, and in the $x-z$ plane (O) at a downstream distance $x / D=0.25$. (b) Power spectral density from a single microphone measurement. (c) Reconstructed pressure fluctuation magnitude (solid line) and experimental measurements at microphone positions $(*) .(d)$ Variation of transverse velocity oscillation over a forcing cycle using reconstructed distribution from pressure measurements (solid line) and spatial averaged component from PIV results (*).

mean normalised streamwise velocity, $\bar{u} / u_{e}$, sampled in both the $y$ and $z$ directions close to the jet exit when the jet is undergoing acoustic forcing. Both velocity profiles correspond to the $x-z$ and $x-y$ planes, respectively. While both profiles are approximately top hat, there is a clear difference between the profiles that can already be observed by comparing the width of the potential core, which is slightly narrower in the $y$ direction. The potential core width was observed to decrease in both profile directions as downstream distance is increased as the shear layers thicken and the jet spreads. However, the spreading rate in the $y$ direction is significantly enhanced due to transverse acoustic forcing, which is discussed further in \$3.2. While the far field is not the focus of the current study, an illustration of this is included in figure 1(c), which shows Mie scattering images of the jet in two planes of interest for both the unforced and forced jet. The application of acoustic forcing is observed to significantly increase the jet spreading rate in the $x-y$ plane and to decrease this in the $x-z$ plane. Although detailed measurements of the far field were not undertaken in this study, the observed increased spreading of the jet is expected to result from the inclination changes in subsequent structures manifested through the induced velocity field, as described by Lee \& Reynolds (1985) and Parekh et al. (1988).

\subsection{Tomographic PIV}

We performed tomographic PIV measurements of the jet using multiple volumetric measurements which were later stitched together. Rather than capture the entire 
volume simultaneously, reducing the depth of the reconstructed slices helped to eliminate ghost particles and to reduce measurement noise (Elsinga et al. 2011). A thick light-sheet was generated using a high-speed laser (Litron LDY303HE) and a series of sheet-forming optics (Thorlabs). The ambient region in the chamber and the jet flow were seeded using olive oil droplets that were produced via a Laskin nozzle. The particle images were captured using four high-speed cameras (Photron SA1.1) that were oriented $12.5^{\circ}$ from each other (see figure $1 b$ ). Due to reflections at the nozzle, data upstream of $x / D=0.20$ could not be collected. The particle image separation time was $\delta t=20 \mu \mathrm{s}$ and the vector field separation time was $1 / 900 \mathrm{~s}$. During each experiment phase-locked data were therefore collected at two points in the oscillation cycle. For each laser sheet position, the experiment was run a total of six times, and before each run a phase offset was adjusted so phase-locked results were recorded for 12 equally spaced positions in the cycle.

The volumetric reconstruction was performed using an in-house tomographic PIV implementation of the MART algorithm (Herman \& Lent 1976). A $42 \mathrm{~mm} \times 22 \mathrm{~mm} \times$ $7 \mathrm{~mm}$ volume was discretised at 20 voxels $\mathrm{mm}^{-1}$ to ensure a pixel-to-voxel ratio of one. The routine applies a multiplicative first guess procedure (Worth \& Nickels 2008) to accelerate convergence, requiring only three MART iterations to be performed. The reconstructed light intensity volumes from 20 phase-locked instantaneous realisations were first summed together before cross-correlation was performed. This sum of volumes procedure is the three-dimensional analogue of the two-dimensional average image method described by Meinhart, Wereley \& Santiago (2000), and its application allows the spatial resolution to be improved, while also improving the reconstruction signal-to-noise ratio.

The intensity volumes were next processed using an in-house cross-correlation algorithm with multi-pass processing, window shift and window deformation developed for volumetric PIV (Lawson \& Dawson 2014). The initial window size for the PIV processing was $64^{3}$ voxels, which was reduced to $48^{3}, 40^{3}$ and finally $32^{3}$ voxels, with $75 \%$ window overlap. Vector validation was performed through a median criterion (Westerweel 1994). For each volumetric slice we processed 20 phase-averaged vector fields (which due to the sum of volumes method is itself an average of 20 instantaneous flow realisations) that are used for the analysis presented in this paper. This process yields the phase-average velocity, $\boldsymbol{u}$, which itself can be represented as the sum of the mean velocity $(\overline{\boldsymbol{u}})$ and the phase-averaged fluctuating component $(\widetilde{\boldsymbol{u}})$, or $\boldsymbol{u}=\overline{\boldsymbol{u}}+\widetilde{\boldsymbol{u}}$. While the phase averaging removes the turbulent fluctuations, $\boldsymbol{u}^{\prime}=0$, it should be noted that the phase-averaged fluctuating velocity component $(\widetilde{\boldsymbol{u}})$ captures most of the vortex structures in the region $x / D<2$ because the turbulent fluctuations are very small here.

After each set of experiments the light-sheet was traversed $5 \mathrm{~mm}$ in the $z$ direction and a total of seven volumetric slices were obtained, with an overlap of $1 \mathrm{~mm}$ between volumes. The vector fields were stitched using linear interpolation to obtain a final volume of dimensions $40 \mathrm{~mm} \times 20 \mathrm{~mm} \times 20 \mathrm{~mm}$ centred on the jet centreline. Finite differencing must be applied to evenly spaced PIV data in order to calculate the velocity gradient tensor. A second-order-accurate least squares differencing scheme was adopted (Raffel et al. 1998), except at the edges where forward and backward differencing was used. Gradient fields were smoothed using a $3^{3}$ element Gaussian smoothing kernel, with a standard deviation of $\sigma=0.65$.

\subsection{Vortex line modelling}

Previous studies have used simple inviscid vortex models to capture the turbulent spectra of a jet (Nickels \& Marusic 2001), and to capture the dynamics leading to 
jet bifurcation in the far field (Leonard 1985). In order to understand the source of the flow-field dynamics in the present study a simple inviscid vortex line model was employed, which was developed previously by Berk \& Ganapathisubramani (2019). The model periodically creates coherent structures based on an initial definition of the structure shape, orientation and circulation. These structures are then advected and allowed to develop downstream to understand primarily the role of the induced velocity. This approach allows us to strengthen our conclusions relating to the geometry and dynamics of the flow field, with comparisons drawn as part of the discussion in the following sections. It should be stressed that the aim of the model was not to capture the formation of coherent structures, but rather to better understand their downstream development as well as the role of the induced velocity in the velocity field statistics.

The initial coherent structure shapes were defined to match the experimentally observed inclined hairpins close to the jet exit. Therefore, the initial shape was modelled on an inclined vortex ring, which is left open at one end to mimic a hairpin structure. Each ring was discretised and modelled as a series of $N_{v}=40$ straight vortex filaments with constant non-dimensional circulation, $\Gamma_{v}^{*}=\Gamma_{v} / u_{e} D=0.5$. Each filament is defined through the location of two end points, with these node locations shared between consecutive filaments. Rings were initialised so that their geometry resembled experimental results, with geometric centres positioned at $x / D=0.25, y / D= \pm 0.08$ and $z / D=0$, with an inclination angle defined by a plane rotated around the $z$ axis by an angle of $\Phi_{v}= \pm 22^{\circ}$. Rings were introduced at a rate of two rings per forcing cycle, and alternating structures were given opposite-signed initial inclination, offset initial transverse positions, and the open end location was switched to the edge which was furthest downstream of the ring in order to match the experimental observations. A constant circulation decay rate, $\mathrm{d} \Gamma_{v}^{*} / \mathrm{d}(t / T)=0.1$, was defined based on the experimental measurements, where $T=1 / f_{f}$ is the period, in order to reduce the influence of structures which break down far downstream and allow the model to rapidly converge. Finally, a Rankine vortex core radius of $R_{\text {core }} / D=0.4$ was chosen empirically to prevent unphysical displacements of individual nodes.

The structures are advected by updating the location of the connecting nodes, which are translated according to the superposition of streamwise mean advection, $\boldsymbol{u}_{s}=\left\{u_{s}, 0,0\right\}$, transversely oscillating periodic, $\boldsymbol{u}_{t}(t)=\left\{0, v_{t}(t), 0\right\}$, and induced, $\boldsymbol{u}_{\boldsymbol{i}}(t)$, velocity components. For simplicity the complexity of the jet structure is not represented directly, and instead a streamwise mean velocity corresponding approximately to the average shear-layer velocity was chosen, $u_{s}=0.7 u_{e}$. The transversely oscillating periodic component is modelled as $v_{t}(t)=-A_{v} \sin \left(2 \pi f_{f} t+\phi_{t}\right)$, where $A_{v}=0.1 u_{e}$ and $\phi_{t}=0.3$ are the amplitude and the phase offset required to replicate the experimentally observed oscillating transverse velocity shown in figure $2(d)$. Vortex structures are created when $t / T=0$ and $t / T=0.5$ to ensure that the transverse location of the structures is correctly replicated by the model. Finally, the induced velocity at a node location $(x, y, z)$ from all filaments $(\boldsymbol{d} s)$ is calculated using the Biot-Savart law, given by

$$
\boldsymbol{u}_{i}(x, y, z)=\frac{\Gamma}{4 \pi} \frac{\boldsymbol{d} \boldsymbol{s} \times \boldsymbol{l}}{|\boldsymbol{l}|\left(\max \left[|\boldsymbol{l}|, R_{\text {core }}\right]\right)^{2}},
$$

where $\boldsymbol{l}$ is the vector from the centre of the segment to the point $(x, y, z)$ and $R_{\text {core }}$ is the Rankine vortex radius. The total advection velocity, $\boldsymbol{u}_{a d v}(t)=\boldsymbol{u}_{s}+\boldsymbol{u}_{t}(t)+\boldsymbol{u}_{i}(t)$, together with a non-dimensional time step of $\Delta t_{\bmod } / T=1 / 48$ is then used to advect 

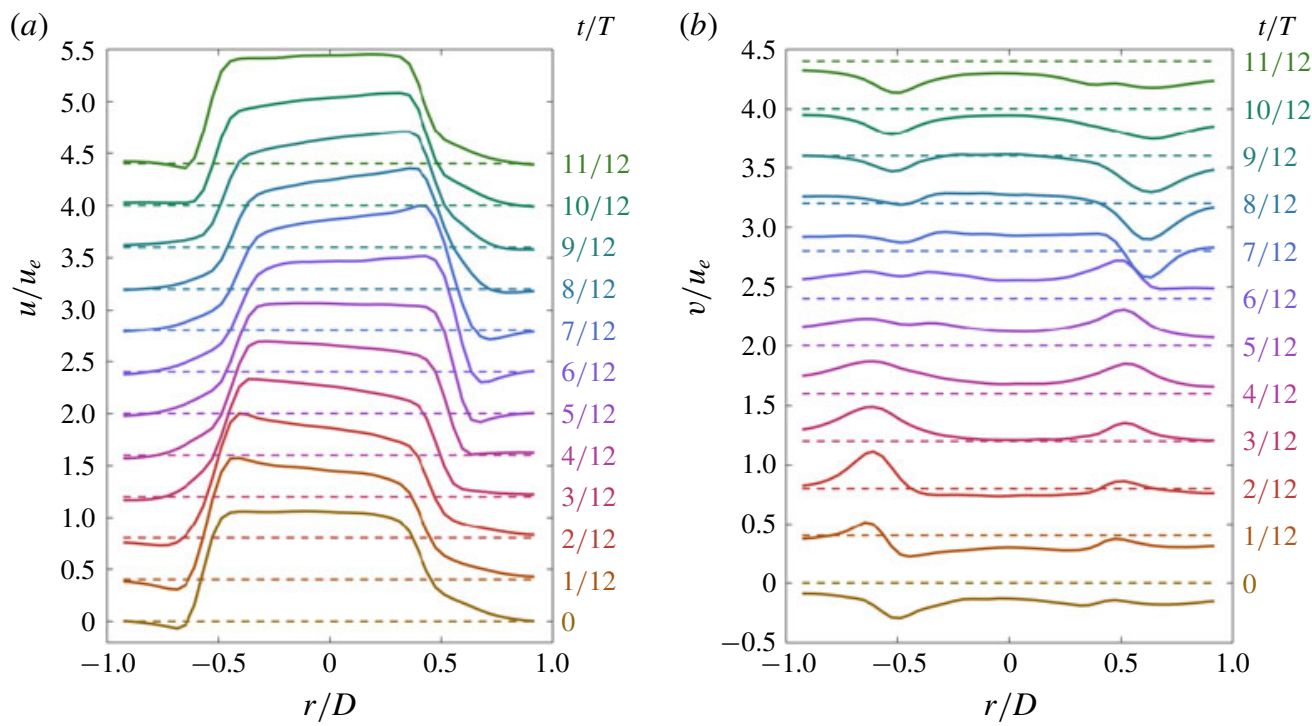

FIGURE 3. Velocity profiles for streamwise and transverse components at each phase angle in the $x-y$ plane, at $x / D=0.25$. Profiles are offset for visibility, with the zero of each profile represented by a dashed line. Lowest profile corresponds to $t / T=0$, with increasing time for each offset curve above.

each node. The model is run for a duration $t / T=10$ to ensure that a steady periodic solution has been reached. On the final cycle the Biot-Savart law is used to calculate the induced velocities on several planes of interest. During velocity field calculation a vector spacing of $\Delta x / D=0.04$ was used, and averaging over a $5 \times 5$ neighbourhood was used in order to match both the experimental spatial resolution and vector pitch. The results of the vortex line modelling are presented and discussed in $\S 3.3$.

\section{Results}

\subsection{Phase-averaged vortex structure}

The jet was positioned at a pressure node of the transverse acoustic mode in the rectangular box. Therefore, the incoming jet flow is not modified by acoustic pressure oscillations in the box, resulting in negligible levels of velocity oscillations in the inlet bulk flow velocity. However, this location is also an acoustic velocity anti-node location. Therefore, the application of acoustic forcing results in a strong transverse flapping motion of the jet at the forcing frequency, which can be viewed as the superposition of both $m= \pm 1$ azimuthal modes. While the acoustic transverse velocity oscillations were described previously in figure $2(d)$, to describe the inlet conditions in more detail the velocity profiles in the $x-y$ plane close to exit are shown in figure 3 .

The phase-averaged exit profiles show a strong dependence on phase angle as expected. Close to the jet axis $(r / D=0)$ the transverse component follows the acoustic velocity, switching sign sinusoidally. The strong peaks and troughs at the jet edges $(r / D \approx \pm 0.5)$ are associated with the induced velocity from the coherent vortex structures located in the shear layers (discussed below). Similar features related to the vortices can also be observed in the streamwise velocity profiles. The changing 
centres of the streamwise profiles additionally demonstrate the flapping motion of the jet, even at this small downstream distance.

In order to understand the structure and phase-averaged dynamics of the jet we begin by studying isosurfaces of normalised vorticity magnitude, $\left|\omega^{*}\right|=|\omega| D / u_{e}$, at three points in the forcing cycle, as shown in figure 4. The isosurfaces have been coloured by the normalised transverse component of vorticity, $\omega_{y}^{*}=\omega_{y} D / u_{e}$, in order to more clearly identify separate structures and to indicate the orientation of vorticity.

As the jet flaps in the transverse direction, vorticity generated in the shear layer is observed to roll up periodically, forming a train of large coherent vortex structures which are advected downstream. The topology of these structures can be described as inverted hairpins (or horseshoes), with the head and legs of each hairpin structure aligned in the cross-stream and transverse directions respectively. The head and legs of a single structure have been marked in figure 4 as B1 and B2, respectively. The term inverted here refers to the legs of each hairpin preceding the head in the streamwise direction. The structures are oriented in this manner at formation due to the inclination of the jet when the roll-up process begins. The orientation of the jet can be observed from the isosurfaces close to the jet exit $(x / D=0)$, showing the orientation of the cylindrical shear layer (marked in figure 4 as A). The orientation can also be inferred from the transverse velocity (shown previously in figure $2 d$ ), which is identified in each panel through the sine wave inset in the upper left corner. It should also be noted that as roll-up occurs when the trajectory of the jet changes direction, two hairpin structures are formed on opposite sides of the jet during each forcing cycle. This behaviour is qualitatively different from that of a jet experiencing purely longitudinal oscillations, a process which results in the formation of a single vortex ring structure during each oscillation cycle (Aydemir et al. 2012). The rapid organisation of the coherent structures in anti-phase with respect to the $x-z$ symmetry plane appears to be qualitatively similar to the alternating pairing structure observed by Urbin \& Metais (1996) and da Silva \& Métais (2002), although in the present investigation this pairing occurs immediately downstream of the exit. This much more rapid organisation is therefore similar to the structures observed by Suzuki et al. (2004).

The transverse flapping motion of the jet also causes the hairpin heads to be located further from the jet centreline in comparison with the legs, resulting in lower advection velocities. This results in an increase in the inclination angle of the hairpin structures with downstream distance from the nozzle due to differences in these advection velocities (with this observation marked $E$ in figure 4). These two observations are quantified in $\S 3.2$. Furthermore, as each hairpin structure advects downstream, the velocity field induced by the head can be observed to draw the legs of the upstream structure through the arch of the hairpin (marked C in figure 4), drawing the two legs of each structure closer together. This process can also be seen to modify the local inclination of the structure. Once they have been threaded through the arches in this manner, the proximity of these leg vortex cores results in the breakdown of the hairpin head, which can then be observed to realign with the legs from the upstream structure (marked $\mathrm{D}$ and $\mathrm{F}$ in figure 4). As the structures advect further downstream $(x / D \geqslant 2)$ this unstable reconnection around the hairpin heads can be observed to result in rapid breakdown of the coherent structure (marked $\mathrm{G}$ in figure 4). The time-dependent formation and downstream evolution of the vortex structures can be more clearly understood from supplementary movies 1 and 2 of normalised vorticity magnitude.

In order to further investigate the position of these vortex structures it is useful to study the components of the vorticity field in different planes of interest. 

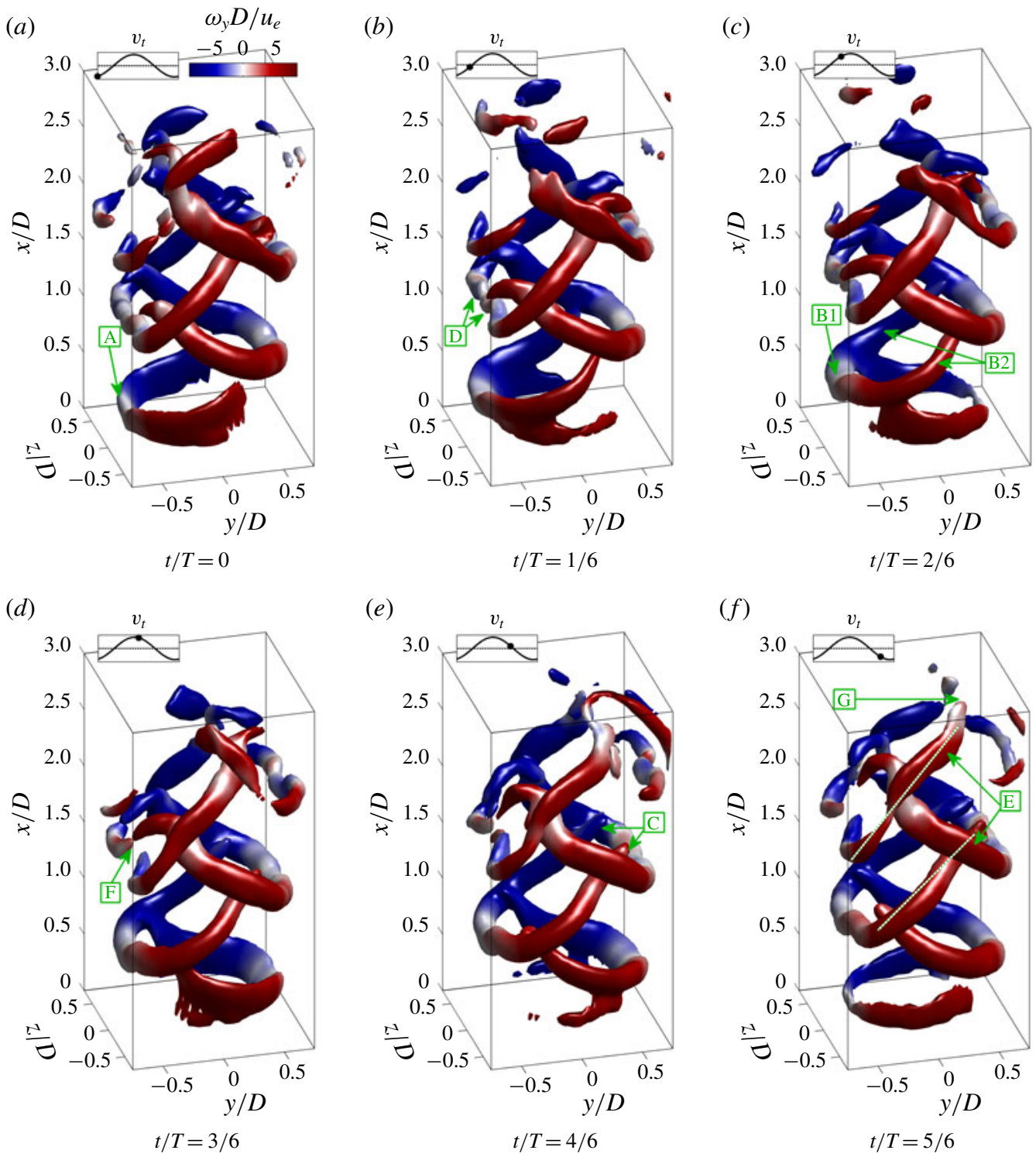

FIGURE 4. Three-dimensional isosurfaces of normalised vorticity magnitude, $\left|\omega^{*}\right|$, at three points in the forcing cycle. A threshold level of $\left|\omega^{*}\right|=6$ was chosen to highlight the vortex structure. The isosurfaces have been coloured by the normalised cross-stream component of vorticity, $\omega_{y}^{*}=\omega_{y} D / u_{e}$, in order to more clearly identify separate structures and to identify the orientation of vorticity. Sine wave inset in upper left corner of each panel indicates the transverse velocity at each phase angle. Annotations identify: A, initial roll-up of the cylindrical vortex sheet shed from the nozzle lip; B1, formation of hairpin head; B2, formation of hairpin legs; C, the legs of the hairpin being drawn through the arch of the preceding structure; D, the formation of oscillations along the head; E, the increasing inclination angle of hairpin structures as they are advected downstream; F, the break-up of the head and reorientation of vortex lines with the legs of the following structure; $\mathrm{G}$, the break-up of coherent structures at the end of the near field. 


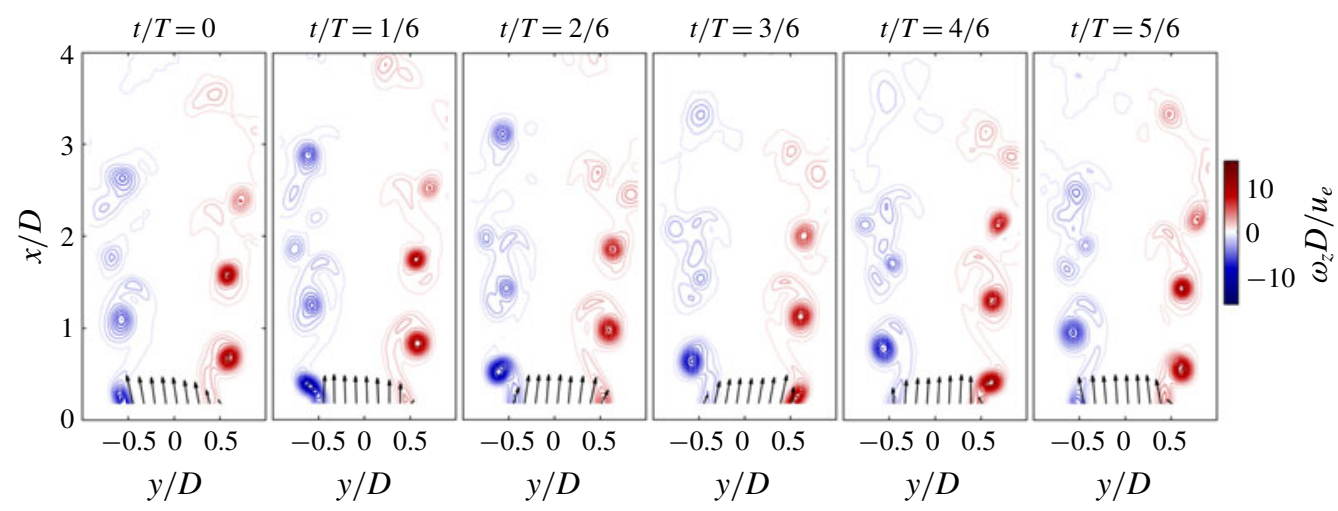

FIGURE 5. Slices of normalised vorticity, $\omega_{z}^{*}=\omega_{z} D / u_{e}$, in the $x-y$ plane, at $z / D=0$, at six points in the forcing cycle. Velocity vectors at the jet exit are included to help identify the corresponding flapping motion of the jet (showing every third vector).

Figure 5 shows the variation of the normalised cross-stream component of vorticity, $\omega_{z}^{*}=\omega_{z} D / u_{e}$, on the $x-y$ plane along the axis of the jet at $z / D=0$, at six instants in the oscillation cycle. This plane of interest therefore cuts through the heads of the inverted hairpin structures, and shows a train of vortex cores of opposite-sign vorticity on the left- and right-hand side of the plot. Close to the exit, structures on the same side of the jet are separated by $\Delta x / D \approx 1$ which corresponds to their advection during a single oscillation cycle at the mean shear-layer velocity, $\left(u_{e} / 2\right) / f_{f} D=1$. However, the downstream location of the vortex cores is staggered between the right- and left-hand sides of the plot, with a difference in axial location of $\Delta x / D \approx 0.5$. This is expected due to the transverse flapping of the jet in response to the acoustic forcing. The formation of structures on the left- and right-hand side of the jet occurs in anti-phase as illustrated by the position of the vortex cores. Close to the jet exit the transverse location of the vortex cores on both sides is $y / D \approx \pm 0.6$, which increases slightly with downstream distance. It is also observed that the flapping of the jet results in an elongated roll-up process. As the jet changes direction the vortex core is located at a larger radial position in comparison with the remaining shear layer, which continues to therefore pass inside of the core. The remaining shear-layer vorticity continues to wrap around and even over the top of the existing vortex core, both increasing its circulation and additionally forming a smaller satellite core downstream. As downstream distance increases the core locations become less distinct as the vortex structure begins to break up, and complex merging and interactions between subsequent structures occur. It should be noted that there is some persistent asymmetry present between structures on the left-hand $(y / D<0)$ and right-hand $(y / D>0)$ sides of the domain. This is ascribed to the imperfect alignment of the jet with the acoustic node position in the enclosure.

The roll-up mechanics is also shown in this plane, and can be described further through a sketch of the process in figure 6, which represents roll-up through the dynamics of the vortex sheet. At $t / T=0$ the magnitude of the transverse acoustic velocity is at a maximum in the negative $y$ direction. As the oscillation cycle progresses, the transverse acoustic velocity changes sign, and in response the jet flaps from its leftward inclined orientation at $t / T=0$, first to slight leftward orientation at $t / T=1 / 6$, and then to a slight rightward inclined orientation at $t / T=2 / 6$. This 


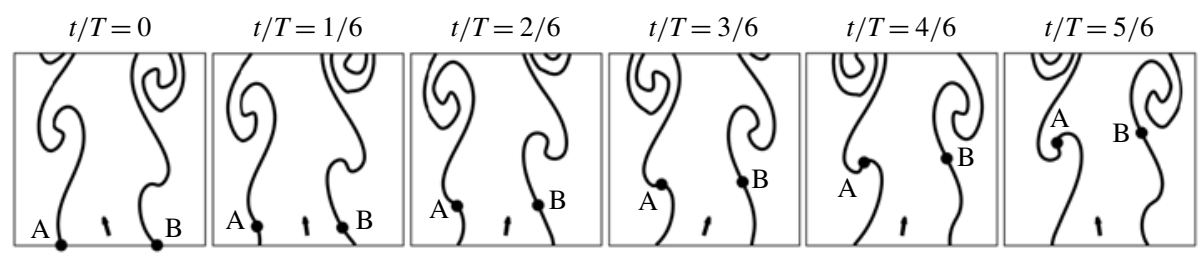

FIGURE 6. Sketch of the vortex sheet roll-up process in the $x-y$ plane. The vector arrow at the bottom centre of each panel represents the jet velocity.

flapping produces an inflection in the vortex sheet, indicated by point A in figure 6 . The sign of the vorticity on the left-hand side is such that the fold in the sheet now begins to roll up under the action of induction, with point $\mathrm{A}$ indicating the rolled-up vortex core head location. During half the oscillation cycle, as the jet flaps from left to right a single hairpin structure is therefore formed. Despite a similar inflection formed through the flapping of the jet, the vortex sheet on the right-hand side of the jet remains stable during the first half of the cycle (shown through a point at a similar height, marked B in figure 6) due to the sign of the vorticity on this side. However, once the jet flaps from right to left to complete a single full oscillation cycle (from $t / T=3 / 6$ to $t / T=0$ ), a second structure is formed on the right-hand side of the jet, due to the now unstable arrangement of the vortex sheet. This roll-up process is therefore distinct from a jet experiencing longitudinal oscillations, in which a variation in circulation along the vortex sheet in the streamwise direction produces roll-up. The current constant circulation vortex sheet roll-up process is driven by the spatial rearrangement of the sheet, through the flapping action of the jet.

Figure 7 shows the variation of the normalised transverse component of vorticity, $\omega_{y}^{*}=\omega_{y} D / u_{e}$, in the $x-z$ plane, at six points in the oscillation cycle. The plane is positioned along the axis of the jet at $y / D=0$, and therefore cuts through the legs of the hairpin structures. In this plane the shear layer is observed to form a train of vortical structures, with structures on either side of the jet centreline having oppositesign vorticity. In contrast with the cross-stream plots (shown in figure 5), the vorticity distribution in the transverse plane is approximately symmetric. Structures at the same downstream location on the left- and right-hand side of the plot correspond to the leftand right-hand legs of a single hairpin structure, which is formed at the same point in the forcing cycle. Given that two hairpin structures are formed during each oscillation cycle, and that each pair of legs can be observed in this plane, the streamwise distance between vortex cores is $\Delta x / D \approx 0.5$. It should also be noted that in comparison with the head locations, the cross-stream location of the vortex cores on both sides of the jet is $z / D \approx \pm 0.5$ close to the jet exit, and decreases slightly with downstream distance.

The symmetry observed in the $x-z$ plane, and the anti-symmetry observed in the $x-y$ plane together with the observations of the three-dimensional structure therefore demonstrate that the current application of transverse acoustic forcing results in the formation of plane-symmetric vortex structures about the $x-z$ plane, which exist with a phase difference of half the forcing cycle. In other words the instantaneous structure of the jet is instantaneously anti-symmetric about the $x-z$ plane, and plane-symmetric in the mean. 


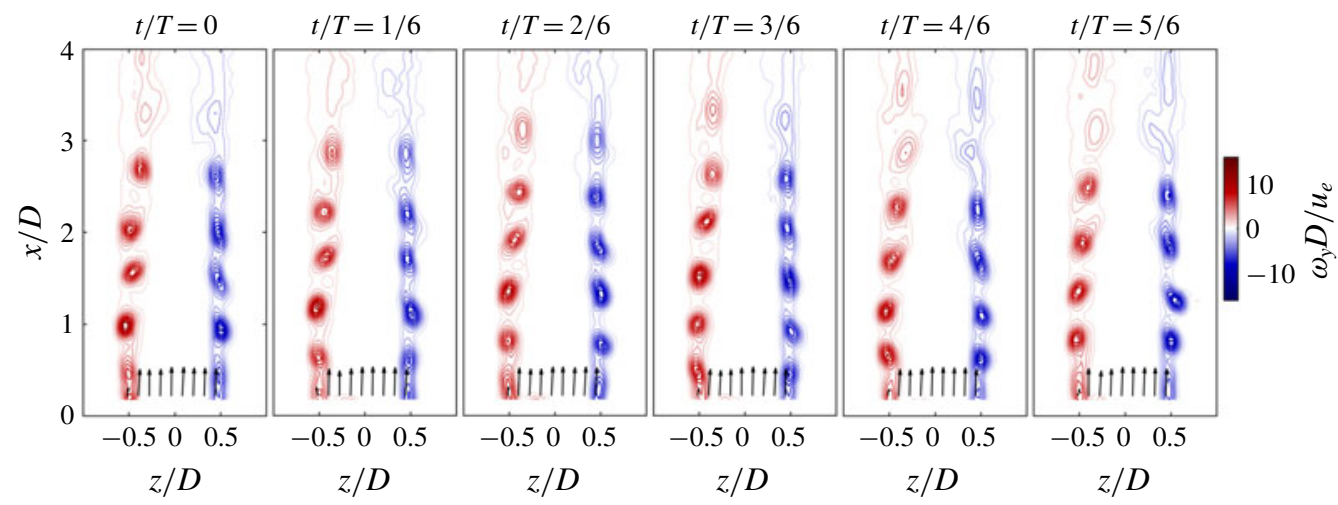

FIGURE 7. Slices of normalised vorticity, $\omega_{y}^{*}=\omega_{y} D / u_{e}$, in the $x-z$ plane, at $y / D=0$, at six points in the forcing cycle. Velocity vectors at the jet exit are included (showing every third vector).

\subsection{Vortex tracking}

In order to quantify the observation that the advection velocity of the hairpin head and legs is different, a simple vortex tracking algorithm was employed. Local vorticity maxima above a threshold value of $\left|\omega^{*}\right|>4$ were located in the jet centreline vorticity slices presented in figures 5 and 7, which allow the in-plane location of the vortex cores described as the hairpin heads and legs to be defined. This choice of slices captures one location for the vortex core of the head, and two locations for the corresponding vortex cores of the legs. A least squares fit to a two-dimensional Gaussian vorticity distribution was performed over a $7 \times 7$ vector window around each of the local maxima in order to determine their locations to sub-vector spacing resolution. The vortex tracking algorithm was applied to each of the phase-averaged fields and the positions of the cores plotted with varying cycle time, as shown in figure $8(a)$. It should be noted that vortex cores formed during four oscillation cycles are simultaneously present in the measurement volume, and therefore the spatial locations of the vortex core positions have been unwrapped with respect to the oscillation cycle to aid the interpretation of their positions. Tracking results from the inviscid vortex model are also plotted in this section, but are discussed further in $\S 3.3$.

Figure 8(a) shows the position of both the vortex core head and legs. Vortex cores associated with structures of opposite orientation are identified separately, with a distinction made between structures with heads lying on either the left-hand $(y / D<0)$ or right-hand $(y / D>0)$ sides of the jet, hereafter referred to as LHS and RHS structures. This separation was made in order to account for the phase difference between structures, allowing a $t / T=-1 / 2$ shift to be applied to RHS structures in order to collapse the data. Despite a small amount of positional scatter, structures on both sides of the jet share similar dynamics. The location of the hairpin head is observed to vary approximately linearly with time, therefore advecting at a near-constant rate. As expected from the inverted orientation of the hairpin structures shown previously, at a given instant in time the hairpin legs are downstream of the heads. Therefore, in figure $8(a)$ the scattered points denoting the hairpin leg locations are located above the head locations. The downstream location of each pair of hairpin legs is shown to match very closely, meaning that the hairpin structures are symmetric 


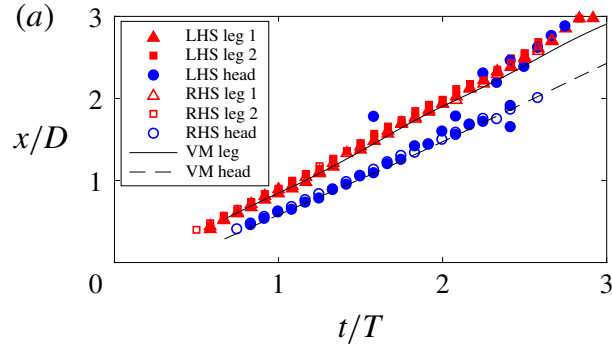

Hairpin vortex core locations

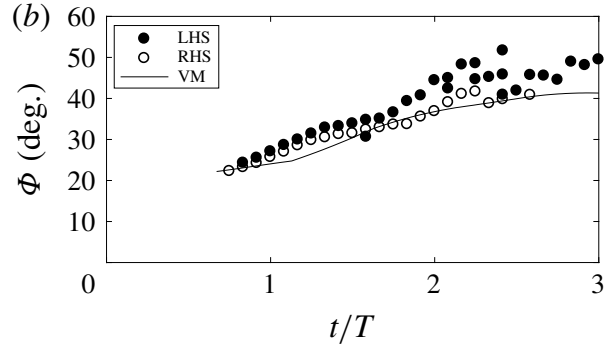

Hairpin inclination angle

FIGURE 8. Vortex core tracking results. In both panels the filled markers show structures with the heads on the left-hand side $(y / D<0)$ and open markers show structures with heads on the right-hand side $(y / D>0)$. (a) Non-dimensional vortex core location against time shows the different advection velocities of cores in the $x-y$ plane (blue circles) and in the $x-z$ plane (red squares and triangles). Solid and dashed lines represent the leg and head positions, respectively, calculated using the vortex model (VM). (b) Variation of the hairpin inclination angle, $\Phi$, with downstream distance. The solid line shows the ring angle calculated using the VM.

about the $x-z$ plane. The location of the legs is also observed to vary approximately linearly with time, again corresponding to a near-uniform advection velocity. However, calculating a linear line of best fit to the core locations demonstrates through the differing slopes that the advection velocities of the head and legs differ, with mean advection velocities of $u_{\text {head }} / u_{e}=0.45$ and $u_{\text {legs }} / u_{e}=0.53$ calculated for the head and legs, respectively.

The difference in advection velocity between the head and legs of each hairpin means that as these structures advect downstream the difference in location between the head and legs increases (as shown by the diverging lines in figure $8 a$ ), meaning the inclination angle of the structures also increases. An estimate of the hairpin inclination angle, $\Phi$, can be made by relating the downstream location of the head and legs at each point in the cycle according to $\Phi=\arctan \left(\left(x_{\text {head }}-x_{\text {legs }}\right) /\left|y_{\text {head }}\right|\right)$. The variation of $\Phi$ with time is shown in figure $8(b)$. The plot shows that immediately after the hairpin structure pinches off, it has an inclination of $\Phi \approx 25^{\circ}$. Based on the magnitude of the transverse acoustic velocity oscillations, the maximum jet angle at exit is approximately $6^{\circ}$. Therefore during the roll-up process, the fluid entering the head and legs of each hairpin structure must also advect at differing velocities, increasing the inclination angle by another $19^{\circ}$ over three-quarters of a cycle. While the rate of this initial inclination increase is not possible to quantify, due to a lack of data at the jet exit, and the difficulty of defining the location of the structure prior to pinch-off, a similar difference in advection rates to the newly pinched-off structure would provide such an increase in inclination over this period. Following pinch-off, the inclination angle is shown to increase with downstream distance with the structure reaching an angle of $\Phi \approx 40^{\circ}$ before breaking up.

The reduced advection velocity of the hairpin heads is due to their repositioning through the action of the jet flapping. This is quantified in figure 9, which shows the variation in radial location of the vortex cores with downstream distance. The markers show that the vortex cores are more widely spread in the $x-y$ plane, with a mean position of $y / D \approx 0.6$, compared with the position of the cores in the $x-z$ plane, which are initially spaced at $z / D \approx 0.5$. However, as shown previously in 


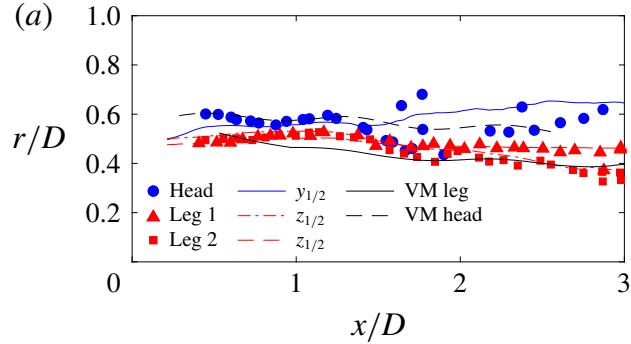

LHS structures $(y / D<0)$ (b)

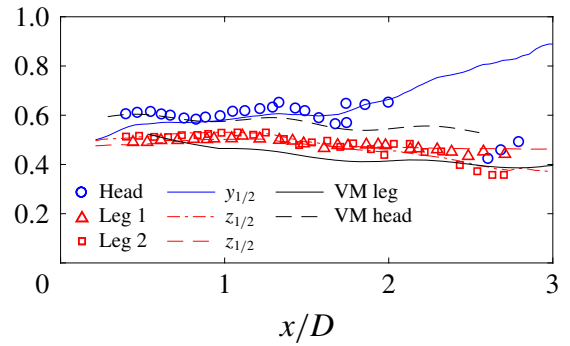

RHS structures $(y / D>0)$

FIGURE 9. Vortex core tracking results showing radial location of vortex cores and shear layer half-width. Both panels show the non-dimensional radial vortex core location against downstream location for vortex cores in the $x-y$ plane (blue circles) and in the $x-z$ plane (red squares and triangles). The mean jet half-widths are also plotted for the $x-y$ plane (solid blue line) and in the $x-z$ plane (dashed red and dash-dot red lines). The radial positions calculated using the vortex model (VM) are also plotted for the head (dashed black line) and legs (solid black line).

figure 2(a), even close to exit, the jet appears to be spreading more rapidly in the $y$ direction due to the transverse acoustic excitation. Therefore it is useful to analyse the location of the vortex cores together with the local spreading rate, defined as the jet half-width $y_{1 / 2}$ in the $x-y$ plane, where $u(y) / u_{e}=1 / 2$, or $z_{1 / 2}$ in the $x-z$ plane, where $u(z) / u_{e}=1 / 2$. In the current study to account for the imperfect alignment of the jet with the acoustic mode, we define the jet half-width separately for leftand right-hand sides on each plane of interest, allowing the vortex location to be compared to the local jet velocity in each case. While the vortices in the $x-z$ plane closely follow the mean shear-layer velocity, the cores the $x-y$ plane periodically fall above this line, and, as a consequence, are advected more slowly downstream. This periodic oscillation in radial location also results in the periodic oscillation in the hairpin inclination angle observed in figure $8(b)$. The jet spreading behaviour is also noteworthy, with a significantly enhanced spreading rate in the transverse direction beyond $x / D>2$, although beyond this location there is considerable asymmetry in the spreading rate on left- and right-hand sides.

The strength and pinch-off time of vortices were characterised through structure circulation in the two planes of interest, and the total circulation present in these planes. This characterisation is similar to the work of Gharib, Rambod \& Shariff (1998), and is calculated here through the integration of the non-dimensional vorticity above a threshold value of $\left|\omega_{i}\right|^{*} \geqslant 1$ over a non-dimensional area, described in the two planes of interest using the following equations:

$$
\begin{aligned}
& \Gamma_{y}^{*}=\int_{x_{1} / D}^{x_{2} / D} \int_{z_{1} / D}^{z_{2} / D} \omega_{y}^{*} \mathrm{~d}\left(\frac{z}{D}\right) \mathrm{d}\left(\frac{x}{D}\right), \\
& \Gamma_{z}^{*}=\int_{x_{1} / D}^{x_{2} / D} \int_{y_{1} / D}^{y_{2} / D} \omega_{z}^{*} \mathrm{~d}\left(\frac{y}{D}\right) \mathrm{d}\left(\frac{x}{D}\right) .
\end{aligned}
$$

The circulation is therefore non-dimensionalised by the jet velocity and diameter, $\Gamma_{i}^{*}=\Gamma_{i} / u_{e} D$. However, the constant circulation generated by the jet shear layer in the current work and the ambiguous pinch-off location of structures in the $x-z$ plane 
at this threshold level required us to use a slightly modified approach which is now described.

In order to neglect circulation from previous cycles, vorticity is only included from a region of interest which is extended at the mean shear-layer velocity. Initially the upper bound of this window, $x_{2}$, was positioned at $x_{0, z} / D=0.2$ in the $x-z$ plane and at $x_{0, y} / D=0.75$ in the $x-y$ plane. The upper-bound location was selected in the $x-y$ plane in order to include the initial shear-layer circulation which never pinches off. Each upper bound was translated downstream at the mean shear-layer velocity, whereas the lower bound was held at $x_{1} / D=0$, thereby extending the region of interest at each time step. The total circulation is assessed between these bound locations, and for leftand right-hand sides of each plane.

In order to assess the circulation contained in the pinched-off structures, separate lower bounds were introduced to separate the contribution from these. The lower vortex bound positions were selected in order to separate the bulk vorticity observed in each structure from that in adjacent structures, and the shear layer. Given that a vortex advecting at the mean shear-layer velocity would translate a non-dimensional distance of $d_{\text {vortex }} / D=\left(u_{e} / 2\right) / f_{f} D=1$ in the current flow, the vortex bound in the $x-y$ plane is set approximately $\Delta x / D=1$ upstream of the upper bound. Two vortex bounds are introduced in the $x-z$ plane to separate the circulation present in the two legs formed during a forcing cycle, with these located at approximately $\Delta x / D=1 / 2$ and $\Delta x / D=1$ upstream of the upper bound. Given that the pinch-off of vortex structures from the shear layer at this threshold level is less obvious than that in previous work (Gharib et al. 1998; Aydemir et al. 2012), and structures in the $x-y$ plane begin to strongly interact with structures from the previous cycle at $t / T=1.3$, these definitions are imperfect. However, separating the circulation in this relatively straightforward manner does allow us to isolate the majority of circulation present in the observed structures, allowing us to make some interesting observations. The bound definitions for all circulation calculations are summarised in the Appendix.

The non-dimensional circulation is plotted in figure 10. The total circulation in both planes is shown to increase approximately linearly with time, which is somewhat expected given the steady generation of vorticity from the jet shear layer. However, in the absence of significant axial velocity oscillations this steady production is significantly different from the behaviour of longitudinally forced jets, where the production of circulation is highly unsteady (Aydemir et al. 2012). The total circulation in the $x-y$ plane does, however, appear to oscillate very slightly, as the transverse velocity appears to first supplement $(t / T<0.5)$ and then counteract $(t / T>0.5)$ the generation of circulation. After the hairpin head has pinched off, its circulation remains relatively constant at $\Gamma_{z}^{*} \approx 1$ for around half a period before interacting with downstream structures. This allows the pinch-off time, where the circulation of the structure matches the total circulation, to be estimated as $t / T \approx 0.5$. This point in the cycle corresponds to transverse fluctuating velocity maxima, or the point at which the jet orientation is rotating away from the forming structure most rapidly. In contrast, the leg structures on the $x-z$ plane contain approximately half the circulation of the heads, $\Gamma_{y}^{*} \approx 0.5$. While this may be obvious from the definition of vortex bounding boxes used, it is a useful point to explicitly confirm, suggesting that each head shares vortex lines, and is thereby linked, not only with its own legs, but also with the legs of the upstream structure. The doubling of the circulation between head and leg structures is therefore consistent with previous visual observations of the complex linking of vortex rings of alternating inclination (Urbin \& Metais 1996; da Silva \& Métais 2002). Finally, it should also be noted that while the head structures contain twice the vorticity of the legs, the spatial organisation of this vorticity (shown previously in figures 5 and 7) is clearly quite different. 


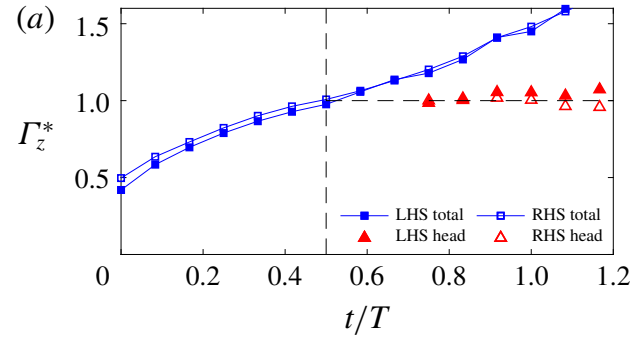

Circulation in $x-y$ plane

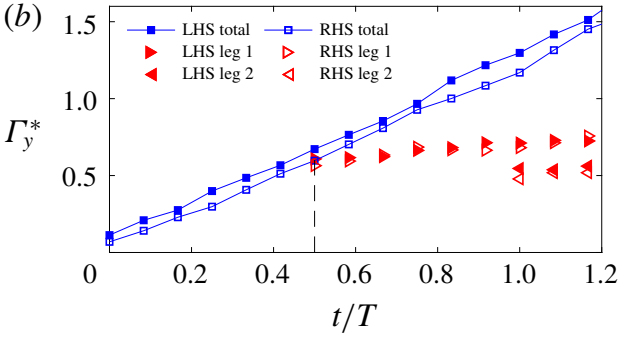

Circulation in $x-z$ plane

FIgure 10. Circulation against time in two planes of interest. Both panels show the total non-dimensional circulation (blue squares). (a) Additionally showing the circulation contained in the hairpin head structures (red triangles). To account for the phase difference between structures on the left- and right-hand sides, a $t / T=-1 / 2$ shift was applied to the structures on the right-hand side, to collapse the data. (b) Additionally showing the circulation contained in the hairpin leg structures (red left-facing and right-facing triangles). In both panels the filled markers show the circulation for structures with heads on the left-hand side $(y / D<0)$ and open markers show structures with heads on the right-hand side $(y / D>0)$.

\subsection{Inviscid vortex line model}

Having experimentally characterised the jet in terms of its dominant vortical structures, it is now useful to present results from the inviscid vortex line model introduced in $\S 2.3$ in order to further understand the role of induction in the development of these structures. Figure 11 shows vortex line representations of structures from the inviscid model, at three points in the forcing cycle. Close to the jet exit, the initial inclined ring shape of the structures is clearly shown, with alternating structures plotted with different colours to aid identification. As the model time advances, these structures are advected downstream with a uniform velocity, as well as advecting in the transverse direction due to the applied periodic acoustic advection velocity. The constant downstream advection results in multiple structures distributed in the streamwise direction, separated as in the experiment by a distance of $\Delta x / D \approx 1$. While difficult to see in this three-dimensional representation due to the smaller transverse component magnitude, the structures also periodically advect back and forth slightly in the transverse direction. However, except for defining the initial relative locations of the structures, these steady and quasi-steady components of the total advection do nothing to modify the shape or orientation of the structures themselves. It is rather the induced velocity component which results in hairpin deformations as they are advected downstream. In order to understand this contribution, velocity vectors representing only the induced velocity component, $\boldsymbol{u}_{i}(t)$, are also plotted at every fourth node location.

As the hairpin structures advect downstream they are observed to deform in a number of ways. In particular, however, there is a strong interaction between the open ends of each ring, which represents the lower part of each hairpin's legs, and the head of the structure immediately downstream. The lower part of each hairpin's legs begins by trailing the head of the structure immediately downstream (A1 in figure 11). However, these are rapidly pulled upwards (A2 in figure 11) and ultimately through the arch and over the top of the head of the structure immediately downstream (A3 and A4 in figure 11), as a result of the induced velocity from the 

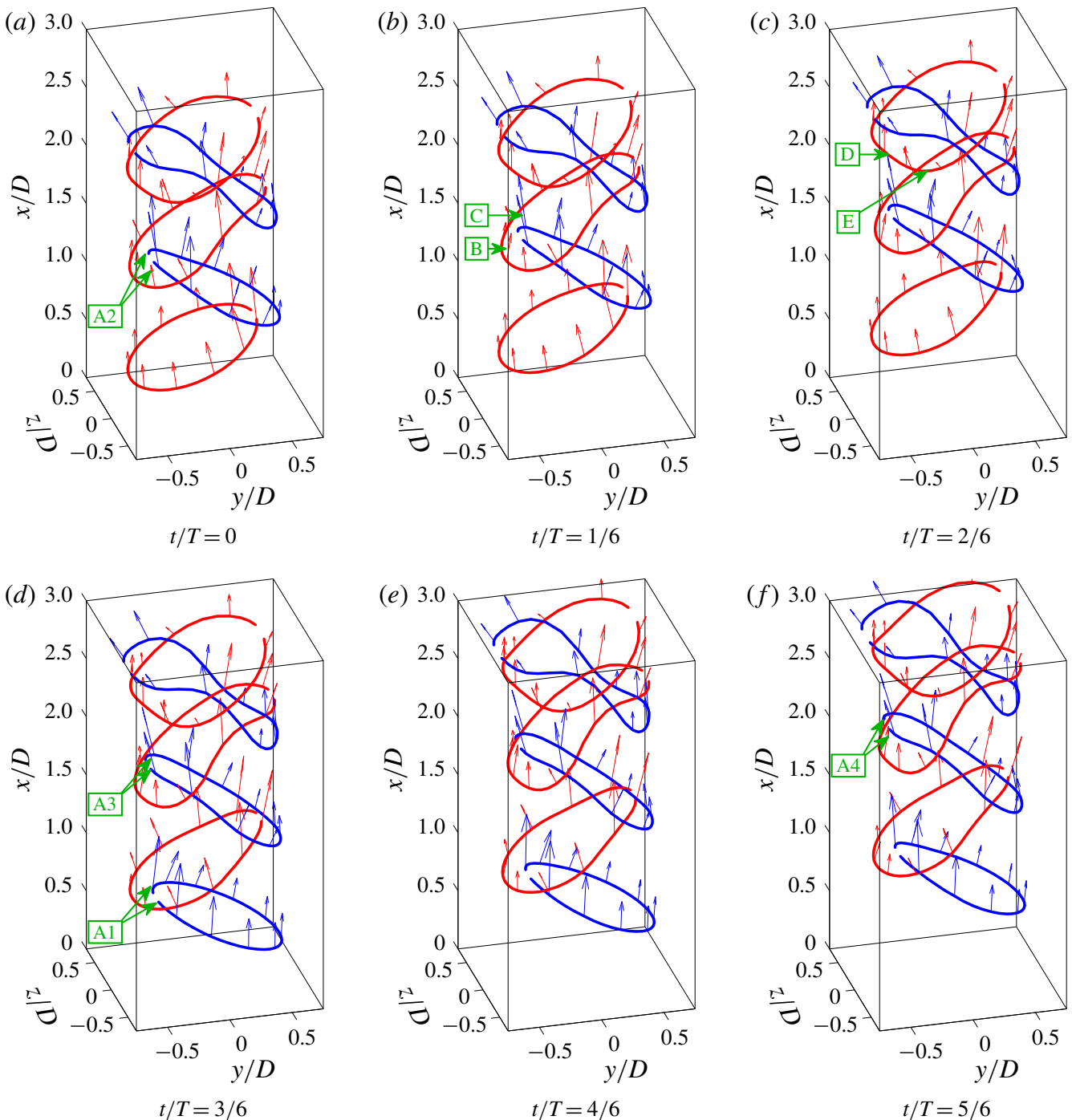

FIGURE 11. Vortex line locations from the inviscid vortex model, at three points in the forcing cycle. Structures with opposite initial inclination angle and orientation are plotted with different colours to aid identification. Velocity vectors representing only the induced component of velocity, $\boldsymbol{u}_{\boldsymbol{i}}(t)$, are also plotted at every fourth node location. Annotations identify: A1-A4, the legs of the hairpin are drawn through the arch of the preceding structure; $\mathrm{B}$, the induced component of velocity on the head has a negative radial component and a modest axial component; $\mathrm{C}$, the induced component on the legs has a positive radial component and a large axial component which in combination give rise to the observed dynamics; D, the centre of the hairpin head begins to arch upward under the induced velocity of the legs; E, the shoulders of the hairpin begin to bow outwards in the cross-stream direction resulting in an increasingly square geometry.

downstream hairpin head. This effect is made apparent when comparing the size and inclination of the velocity vectors on the legs of one structure and the head of the downstream structure. Higher velocity magnitudes and a positive radial component 
are observed on the lower part of the legs ( $\mathrm{C}$ in figure 11), and lower velocity magnitudes and a negative radial component are seen on the heads ( $\mathrm{B}$ in figure 11).

The lower part of the hairpin legs also induces a reciprocal effect, causing the head of the downstream structure to arch upwards in its centre (D in figure 11), and the shoulders of the structure (the points approximately between the head and legs) to bow downwards in the streamwise and outwards in the cross-stream direction ( $\mathrm{E}$ in figure 11). The deformation this induces bears strong similarities to the structures observed experimentally in figure 4 . Therefore, while this simple model cannot capture the full complexity of the jet dynamics, it does indicate that the increasingly square geometry of the structures as they advect downstream, the increasing hairpin angle and the deformation and eventual break-up of the structure head may all be a result of the interaction between subsequent structures through mutual induction.

The structure dynamics predicted by the vortex model can be further quantified by examining the downstream and radial locations of the filaments and the corresponding evolution of the hairpin inclination angle. The normalised hairpin head and leg locations were defined in a manner similar to those for the experimental results, in this case through the intersection of the closest vortex filament from each structure with the $x-y$ and $x-z$ planes for the head and legs, respectively. The inclination angles were also defined in a similar manner, and calculated from these locations. In order to compare directly with the experimental results, the locations and angles from the vortex model were plotted previously in figures 8 and 9, and are now discussed.

As observed previously in the experimental results, figure 9 shows that the heads are created at a larger radius than the legs due to the transverse flapping of the jet. In the model the radial positioning of the hairpin heads at small downstream distances is correctly replicated primarily through the initialisation of the ring position. The periodic oscillation of head radial position with downstream location is also well captured at moderate downstream distances, through the specified phase relationship of the transverse acoustic advection velocity.

As shown in figure 9, the legs are initially located at smaller radii in comparison with the heads, and as the structures advect downstream they are seen to move further inwards radially. This inward radial movement of the legs is captured correctly by the vortex model which also shows a decrease in radial position with increasing downstream distance. This behaviour in the modelled structures can be attributed to the interaction induced between subsequent structures. As the two legs from a hairpin structure are pulled up and through the head of the downstream structure, they are induced to bunch more closely together. As the hairpin legs bunch together they induce greater velocities on one another and advect more rapidly downstream. Conversely the larger radial location of the hairpin heads means that these experience lower induced velocities, and therefore advect more slowly downstream. In conformity with the experimental results, these differing advection velocities are reflected in both the downstream locations of the head and legs shown in figure $8(a)$, and also the vortex inclination angles calculated from these in figure $8(b)$. Therefore, despite its simplistic formulation, the vortex model provides reasonable prediction of the development of the vortex locations in the near field, indicating that the complex dynamics of the hairpin structures is largely driven by induction. The model cannot, however, capture the break-up of the vortices, and therefore the radial locations of the head at large downstream distances are poorly described.

\subsection{Root mean square velocity oscillations}

It is also interesting to examine the velocity field statistics through the root mean square (RMS) velocity oscillations. On an unforced turbulent round jet these would be 
(a)

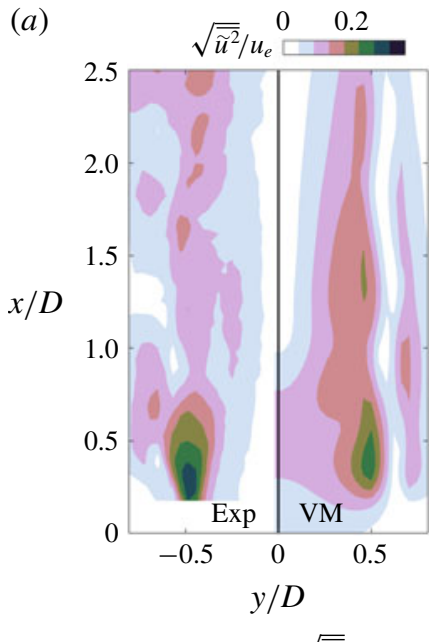

(d)

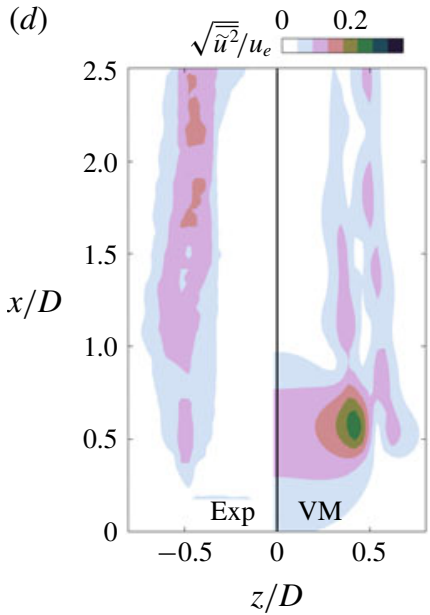

$x-z$ plane, $\sqrt{\overline{\bar{u}^{2}}} / u_{e}$ (b)

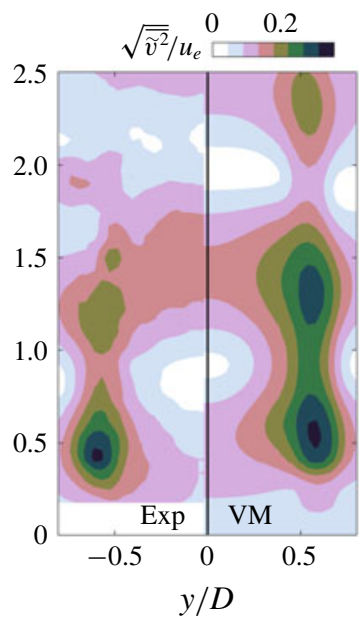

(e)

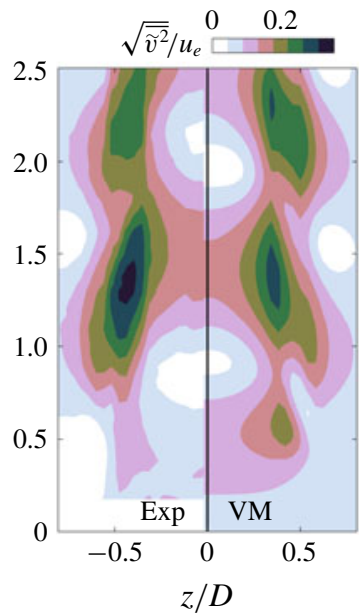

$x-z$ plane, $\sqrt{\overline{\widetilde{v}^{2}}} / u_{e}$ (c)

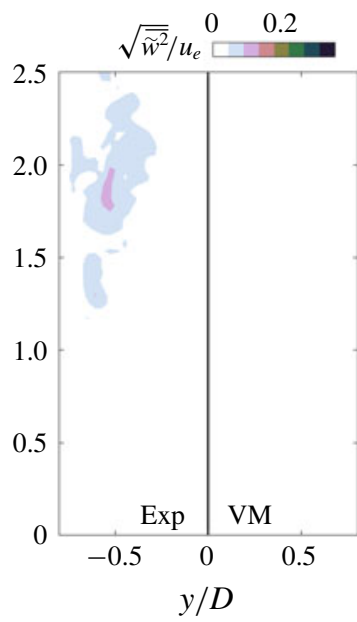

$x-y$ plane, $\sqrt{\overline{\widetilde{w}^{2}}} / u_{e}$

(f)

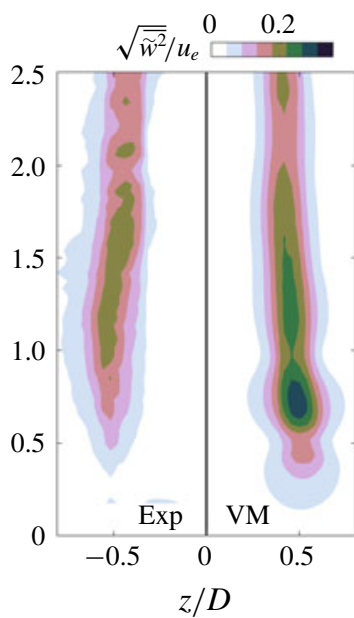

$x-z$ plane, $\sqrt{\overline{\widetilde{w}^{2}}} / u_{e}$

FIGURE 12. Comparison of normalised components of the RMS velocity between experimental (Exp.) and vortex model (VM) results. Contours are presented in both the $x-y$ plane at $z / D=0$ and the $x-z$ plane at $y / D=0$.

expected to grow in amplitude with downstream distance as the shear layers develop, reaching modest amplitudes (see for example Pope (2000), §5.4.1, p. 136). However, the formation of large-scale phase-averaged vortex structures at the jet exit due to acoustic forcing significantly modifies their distribution. To illustrate this, normalised RMS velocity oscillations are plotted in figure 12 on both the $x-y$ and $x-z$ planes from both the experiment (left-hand half of each panel) and the model (right-hand half of each panel). It should be noted that both experimental and model results lack a turbulent fluctuating component, and therefore the RMS components in the present study are representative of the phase-averaged velocity fluctuations, $\sqrt{\overline{\widetilde{\boldsymbol{u}}^{2}}}$, rather than 
the more conventional sum of phase-averaged fluctuating and turbulent oscillations, $\sqrt{\overline{\left(\widetilde{\boldsymbol{u}}+\boldsymbol{u}^{\prime}\right)^{2}}}$.

The streamwise component of the RMS velocity, $\sqrt{\overline{\widetilde{u}^{2}}} / u_{e}$, is presented in figures $12(a)$ and $12(d)$ for the $x-y$ and $x-z$ planes, respectively. The $x-y$ plane captures the transverse jet oscillation, and the experimental results show a highmagnitude region close to the jet exit and a lower-magnitude streak which follows the meandering trajectory of the vortex structures as these advect downstream. In the $x-z$ plane there are no large oscillations at the base of the jet in the experimental results, but a similar lower-magnitude streak appears along the shear layer, with the shape of this streak again closely following the vortex trajectories observed previously. It is also worth commenting on the magnitude of oscillations. Experimentally RMS velocities are observed up to approximately $30 \%$ of the bulk flow velocity, which is significantly larger than the magnitude of turbulent fluctuations in the near field of an unforced jet.

The model results in the right-hand half of the panels of figure $12(a, d)$ show artificially high regions of oscillating velocity in the near field close to the jet axis in both planes of interest. These regions result from the artificial method of structure formation used in the current model. The sudden placement of structures, rather than directly modelling the roll-up process, gives rise to large oscillations over the forcing cycle close to the jet exit. However, as our current interest is in understanding structure development, accepting this deficiency the model does appear to capture some important features of the downstream RMS distribution, replicating to a certain degree the high-magnitude streaks associated with the meandering path of the vortex cores, and the approximate magnitude of the RMS velocity.

The transverse component of oscillating velocity, $\sqrt{\widetilde{\widetilde{v}}^{2}} / u_{e}$, is shown in figures $12(b)$ and $12(e)$ for the $x-y$ and $x-z$ planes, respectively. From the experimental results, the fields are dominated by a series of high-magnitude regions, which again follow the trajectory of the vortex cores. The pinching and expanding of these high-magnitude regions in the $x-y$ plane with increasing downstream distance result from the combination of the induced velocity from the vortex cores either combining with or cancelling out the transverse acoustic velocity oscillations. This results in a distinctive patternation of the field, the essential features of which are replicated relatively well by the vortex model. However, the vortex model is not capable of accurately capturing the vortex breakdown, and therefore larger discrepancies are seen with increasing downstream distance $(x / D>2)$. Similarly, important features of the transverse component in the $x-z$ plane, such as the inwardly inclined high-magnitude streaked regions close to the jet shear layers and the low-magnitude elliptical regions along the jet centreline, are also represented relatively well by the vortex model in the near field.

Finally, reasonable agreement in the distribution and amplitude of RMS velocity is also observed for the cross-stream component, $\sqrt{\overline{\widetilde{w}^{2}}} / u_{e}$, as shown in figure $12(c, f)$.

The reasonably good agreement between experimental and numerical results in terms of both distribution and amplitude of velocity oscillations demonstrates that the velocity field statistics are dominated by the large-scale hairpin structures, with the pronounced departures from the behaviour of an unforced jet driven by velocity induced by the coherent hairpin structures in addition to the contribution from the acoustic field, rather than by jet instabilities or other viscous effects. 


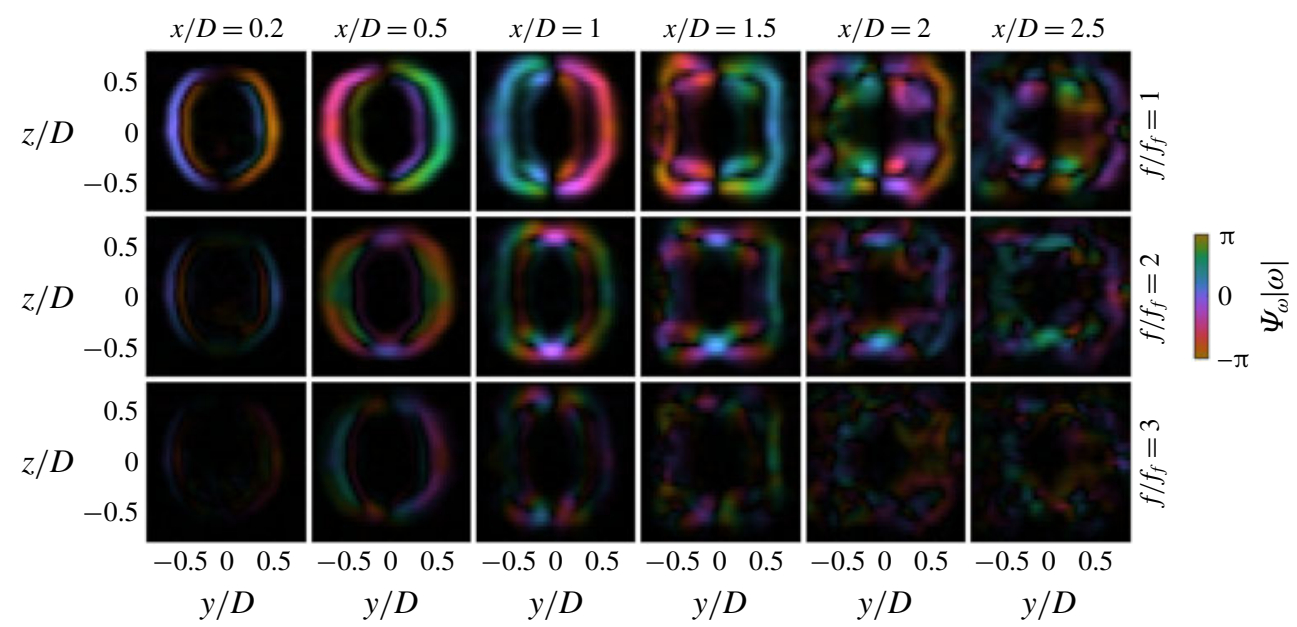

FIGURE 13. Weighted phase of vorticity fluctuations in the $y-z$ plane at different downstream locations, showing the response at the forcing frequency and second and third harmonics. The colour in each panel represents the phase angle of the fluctuation at the measurement location, and the brightness represents the oscillation magnitude.

\subsection{Spectral analysis}

In order to further analyse the spectral content of the velocity gradient field, the weighted phase of vorticity fluctuations was calculated in $y-z$ planes at different downstream locations, shown in figure 13. The weighted phase calculation used an approach similar to that of Hauser et al. (2011), in which the fast Fourier transform of the vorticity magnitude fluctuation at each measurement point within each plane of interest was taken, before using these complex values to calculate the magnitude, $|\omega(f)|$, and phase, $\Psi_{\omega}(f)$, of vorticity oscillations for different harmonic components ( $f=m f_{f}$, where $m$ is an integer). Weighted phase images were created at integer values of the forcing frequency by using colour to denote the phase angle of the oscillations at each measurement location, and brightness to denote amplitude. Through this weighting the phase variation is only discernible in regions with a significant fluctuation amplitude, providing a useful way of understanding the most significant vorticity oscillations. Zero padding was used in order to ensure frequency bins were located at integer multiples of the forcing frequency, and Hann windowing was employed in order to reduce dependence on the limited sample size. Finally, it is also important to note that the cyclic colour map was selected to be perceptibly uniform prior to the brightness weighting process (Thyng et al. 2016).

The development of the vortex structures as they advect downstream can be understood by looking from left to right on the figure. Images in the top row are brightest, indicating that the vorticity field is dominated by oscillations at the first harmonic. Crescent-shaped regions in this row correspond to the head and legs of the vortex structures, which are clearly visible on each side of the $y$ axis. The phase colouring shows these structures are in anti-phase with each other at each downstream location, corresponding to the previously observed staggered downstream locations of the vortex core heads. As the hairpin structures are advected downstream they can be seen to deform, from initially round close to the jet exit to increasingly square. It is also interesting to note that immediately downstream of the jet exit, 
vorticity fluctuations along structures either side of the $y$ axis are in phase, but as these structures are advected downstream, they exhibit phase variations along each structure. These waves are likely to arise as a result of the observed mutual interaction of the structures with vortices downstream.

While vorticity fluctuations at the second harmonic are in general significantly lower in amplitude in comparison with the fundamental frequency, the middle row of weighted phase images is marked by two bright points between $1<x / D<2$, which lie on the $y$ axis at $x / D= \pm 0.5$. These two points represent the passage of the hairpin leg structures, but these peaks are now centred at $f / f_{f}=2$, due to the formation of two structures every forcing cycle. The way that these hairpin structures are interwoven means that only a relatively small region of the flow experiences a strong frequency doubling due to the leg vortices advecting past the same volumetric location. The figure also demonstrates how short-lived these structures are, with the magnitude of the other harmonic components becoming similar to that of the principal components further downstream, $x / D \geqslant 2.5$, which is indicative of their rapid subsequent breakdown.

\section{Conclusions}

In the present paper we have studied a turbulent jet subject to transverse acoustic excitation using tomographic PIV. The jet was located at the pressure node location in an enclosure, and experienced a strong transverse flapping motion which was found to dominate its structure and dynamics. The vorticity field was dominated by the roll-up and advection of inverted hairpin structures, with two of these structures formed during each forcing cycle. The vorticity distribution was found to be plane-symmetric with a half-cycle offset in phase, and could therefore be described as anti-symmetric about the $x-z$ plane. This anti-symmetric distribution of vorticity represents a significant departure from the structures of axially forced jets, which are dominated by unsteady axisymmetric structures.

As the hairpin structures advect downstream their orientation angle was shown to increases due to different advection velocities of head and leg vortices. These velocity differences are caused by the spatial organisation of the vortices as a result of the roll-up mechanics. The flapping motion of the jet causes the hairpin heads to be formed at larger radial distances in comparison with the hairpin legs, resulting in a reduction in their induced advection velocity. Furthermore as the structures are advected downstream they were observed to interact through mutual induction. These effects resulted in the distortion of the hairpin shape, meaning they became increasingly square with downstream distance, and also increasingly intertwined.

The formation of two distinct structures during each forcing cycle results in the generation of harmonic oscillations at certain locations in the volume. The hairpin structures were shown to be short-lived, rapidly breaking down after several jet diameters, which was accompanied by rapid jet spreading in the transverse plane. A simple vortex model was introduced, and it was found that the vortex dynamics and distribution of velocity fluctuations in the flow are dominated by velocity induced by the phase-averaged large-scale structures.

\section{Acknowledgements}

N.A.W. would like to acknowledge support from the European Research Council (ERC) under the European Union's Horizon 2020 research and innovation programme (grant agreement no. 677931 TAIAC). The authors would also like to thank E. Æsøy for supplying jet images. 


\section{Supplementary movies}

Supplementary movies are available at https://doi.org/10.1017/jfm.2019.821.

\section{Appendix}

Tables 1 and 2 describe the bound positions for the calculation of circulation in the heads, legs and total domain as described in $\S 3.2$.

$$
\begin{array}{ccccc} 
& x_{1} / D & x_{2} / D & y_{1} / D & y_{2} / D \\
\Gamma_{z}^{*} \text { total LHS } & 0 & \frac{x_{0, z}}{D}+\frac{t}{T} & -1 & 0 \\
\Gamma_{z}^{*} \text { total RHS } & 0 & \frac{x_{0, z}}{D}+\frac{t}{T}-\frac{1}{2} & 0 & 1 \\
\Gamma_{z}^{*} \text { head LHS } & \frac{x_{2}}{D}-1 & \frac{x_{0, z}}{D}+\frac{t}{T} & -1 & 0 \\
\Gamma_{z}^{*} \text { head RHS } & \frac{x_{2}}{D}-1 & \frac{x_{0, z}}{D}+\frac{t}{T}-\frac{1}{2} & 0 & 1
\end{array}
$$

TABLE 1. Bound values for $\Gamma_{z}^{*}$ circulation calculations in $\$ 3.2$, based on an initial bound of $x_{0, z} / D=0.2$. The bounds are time dependent. Calculation of the LHS starts at $t / T=0$, whereas calculation of the RHS starts at $t / T=1 / 2$.

$$
\begin{array}{ccccc} 
& x_{1} / D & x_{2} / D & z_{1} / D & z_{2} / D \\
\Gamma_{y}^{*} \text { total LHS } & 0 & \frac{x_{0, y}}{D}+\frac{t}{T} & -1 & 0 \\
\Gamma_{y}^{*} \text { total RHS } & 0 & \frac{x_{0, y}}{D}+\frac{t}{T}-\frac{1}{2} & 0 & 1 \\
\Gamma_{y}^{*} \text { leg 1 LHS } & \frac{x_{2}}{D}-\frac{1}{2} & \frac{x_{0, y}}{D}+\frac{t}{T} & -1 & 0 \\
\Gamma_{y}^{*} \operatorname{leg} 1 \text { RHS } & \frac{x_{2}}{D}-\frac{1}{2} & \frac{x_{0, y}}{D}+\frac{t}{T}-\frac{1}{2} & 0 & 1 \\
\Gamma_{y}^{*} \operatorname{leg} 2 \text { LHS } & \frac{x_{2}}{D}-1 & \frac{x_{0, y}}{D}+\frac{t}{T}-\frac{1}{2} & -1 & 0 \\
\Gamma_{y}^{*} \operatorname{leg} 2 \text { RHS } & \frac{x_{2}}{D}-1 & \frac{x_{0, y}}{D}+\frac{t}{T}-1 & 0 & 1
\end{array}
$$

TABLE 2. Bound values for $\Gamma_{y}^{*}$ circulation calculations in $\S 3.2$, based on an initial bound of $x_{0, y} / D=0.75$. The bounds are time dependent. Calculation of the LHS starts at $t / T=0$, whereas calculation of the RHS starts at $t / T=1 / 2$.

\section{REFERENCES}

Aydemir, E., Worth, N. A. \& DaWson, J. R. 2012 The formation of vortex rings in a strongly forced round jet. Exp. Fluids 52 (3), 729-742.

Bauerheim, M., Salas, P., Nicoud, F. \& Poinsot, T. 2014 Symmetry breaking of azimuthal thermo-acoustic modes in annular cavities: a theoretical study. J. Fluid Mech. 760, 431-465.

Becker, H. A. \& MASSARo, T. A. 1968 Vortex evolution in a round jet. J. Fluid Mech. 31 (3), $435-448$. 
Berk, T. \& GAnApathisubramani, B. 2019 Effects of vortex-induced velocity on the development of a synthetic jet issuing into a turbulent boundary layer. J. Fluid Mech. 870, 651-679.

Bourgouin, J. F., Durox, D., Moeck, J. P., Schuller, T. \& CAndel, S. 2015 Characterization and modeling of a spinning thermoacoustic instability in an annular combustor equipped with multiple matrix injectors. Trans. ASME J. Engng Gas Turbines Power 137 (2), 021503.

Crow, S. C. \& Champagne, F. H. 1971 Orderly structure in jet turbulence. J. Fluid Mech. 48, 547-591.

Danaila, I. \& Boersma, B. J. 2000 Direct numerical simulation of bifurcating jets. Phys. Fluids 12 (5), 1255-1257.

DAwson, J. R. \& WorTh, N. A. 2014 Flame dynamics and unsteady heat release rate of self-excited azimuthal modes in an annular combustor. Combust. Flame 161 (10), 2565-2578.

DAwson, J. R. \& WoRTh, N. A. 2015 The effect of baffles on self-excited azimuthal modes in an annular combustor. Proc. Combust. Inst. 35 (3), 3283-3290.

Dowling, A. P. 1997 Nonlinear self-excited oscillations of a ducted flame. J. Fluid Mech. 346, 271-290.

Elsinga, G. E., Westerweel, J., Scarano, F. \& Novara, M. 2011 On the velocity of ghost particles and the bias errors in Tomographic-PIV. Exp. Fluids 50 (4), 825-838.

Gharib, M., RAmbod, E. \& SharifF, K. 1998 A universal time scale for vortex ring formation. J. Fluid Mech. 360, 121-140.

Ghirardo, G., Juniper, M. P. \& Moeck, J. P. 2016 Weakly nonlinear analysis of thermoacoustic instabilities in annular combustors. J. Fluid Mech. 805, 52-87.

Gohil, T. B., Saha, A. K. \& Muralidhar, K. 2015 Simulation of the blooming phenomenon in forced circular jets. J. Fluid Mech. 783, 567-604.

Gutmark, E. \& Ho, C.-M. 1983 Preferred modes and the spreading rates of jets. Phys. Fluids 26 (10), 2932-2938.

Hauser, M., Lorenz, M. \& SAttelmayer, T. 2011 Influence of transversal acoustic excitation of the burner approach flow on the flame structure. Trans. ASME J. Engng Gas Turbines Power 133 (4), 041501.

Herman, G. T. \& Lent, A. 1976 Iterative reconstruction algorithms. Comput. Biol. Med. 6 (4), 273-294.

Ho, C.-M. \& Huerre, P. 1984 Perturbed free shear layers. Annu. Rev. Fluid Mech. 16 (1), $365-422$.

Hussain, A. K. M. F. \& ZAMAN, K. B. M. Q. 1980 Vortex pairing in a circular jet under controlled excitation. Part 2. Coherent structure dynamics. J. Fluid Mech. 101 (3), $493-544$.

JANG, S.-H. \& IH, J.-G. 1998 On the multiple microphone method for measuring in-duct acoustic properties in the presence of mean flow. J. Acoust. Soc. Am. 103 (3), 1520-1526.

KASAGI, N. 2006 Toward smart control of turbulent jet mixing and combustion. JSME Intl J. 49 (4), 941-950.

LAwson, J. M. \& DAwson, J. R. 2013 The formation of turbulent vortex rings by synthetic jets. Phys. Fluids 25 (10), 105113.

LAwson, J. M. \& DAWson, J. R. 2014 A scanning PIV method for fine-scale turbulence measurements. Exp. Fluids 55, 1857.

LEe, M. \& Reynolds, W. C. 1985 Bifurcating and blooming jets at high Reynolds number. In Fifth Symposium on Turbulent Shear Flows, Ithaca, New York, 1.7-1.12.

LEONARD, A. 1985 Computing three-dimensional incompressible flows with vortex elements. Annu. Rev. Fluid Mech. 17 (1), 523-559.

Lespinasse, F., Baillot, F. \& Boushaki, T. 2013 Responses of V-flames placed in an hf transverse acoustic field from a velocity to pressure antinode. C. R. Méc 341 (1-2), 110-120.

Longmire, E. K. \& Duong, L. H. 1996 Bifurcating jets generated with stepped and sawtooth nozzles. Phys. Fluids 8 (4), 978-992.

Meinhart, C. D., Wereley, S. T. \& Santiago, J. G. 2000 A PIV algorithm for estimating time-averaged velocity fields. Trans. ASME J. Fluids Engng 122 (2), 285-289.

NiCKELS, T. B. \& MARUSIC, I. 2001 On the different contributions of coherent structures to the spectra of a turbulent round jet and a turbulent boundary layer. J. Fluid Mech. 448, 367-385. 
O'Connor, J. \& LIEuwen, T. 2011 Disturbance field characteristics of a transversely excited burner. Combust. Sci. Technol. 183 (5), 427-443.

O'Connor, J. \& Lieuwen, T. 2012 Recirculation zone dynamics of a transversely excited swirl flow and flame. Phys. Fluids 24 (7), 2893-2900.

Parekh, D. E., Leonard, A. \& Reynolds, W. C. 1988 Bifurcating jets at high Reynolds numbers. Tech. Rep. Stanford University, Department of Mechanical Engineering.

Pope, S. B. 2000 Turbulent Flows. Cambridge University Press.

Raffel, M., Willert, C., Wereley, S. \& Kompenhans, J. 1998 Particle Image Velocimetry, 2nd edn. Springer.

Reynolds, W. C., Parekh, D. E., Juvet, P. J. D. \& Lee, M. J. D. 2003 Bifurcating and blooming jets. Annu. Rev. Fluid Mech. 35 (1), 295-315.

SADEGHI, H. \& PollaRd, A. 2012 Effects of passive control rings positioned in the shear layer and potential core of a turbulent round jet. Phys. Fluids 24 (11), 105103.

DA Silva, C. B. \& MÉTAis, O. 2002 Vortex control of bifurcating jets: a numerical study. Phys. Fluids 14 (11), 3798-3819.

SUZUKI, H., KASAGi, N. \& SUZUKI, Y. 2004 Active control of an axisymmetric jet with distributed electromagnetic flap actuators. Exp. Fluids 36 (3), 498-509.

Thyng, K. M., Greene, C. A., Hetland, R. D., Zimmerle, H. M. \& Dimarco, S. F. 2016 True colors of oceanography: guidelines for effective and accurate colormap selection. Oceanography 29 (3), 9-13.

Trouvé, A. \& Poinsot, T. 1994 The evolution equation for the flame surface density in turbulent premixed combustion. J. Fluid Mech. 278, 1-31.

TyliszCZaK, A. 2015 Multi-armed jets: A subset of the blooming jets. Phys. Fluids 27 (4), 041703.

TYliszCZAK, A. \& GeURTS, B. J. 2014 Parametric analysis of excited round jets-numerical study. Flow Turbul. Combust. 93 (2), 221-247.

Urbin, G. \& Metais, O. 1996 Large-eddy simulations of three-dimensional spatially developing round jets. In Direct and Large Eddy Simulations II (ed. J.-P. Chollet, P. R. Voke \& K. Leonhard), Springer.

WESTERWEEL, J. 1994 Efficient detection of spurious vectors in particle image velocimetry data. Exp. Fluids 16 (3), 236-247.

Wolf, P., Staffelbach, G., Gicquel, L. Y. M., Müller, J. D. \& Poinsot, T. 2012 Acoustic and large eddy simulation studies of azimuthal modes in annular combustion chambers. Combust. Flame 159 (11), 3398-3413.

Worth, N. A. \& Dawson, J. R. 2013 Modal dynamics of self-excited azimuthal instabilities in an annular combustion chamber. Combust. Flame 160 (11), 2476-2489.

Worth, N. A. \& Nickels, T. B. 2008 Acceleration of Tomo-PIV by estimating the initial volume intensity distribution. Exp. Fluids 45 (5), 847.

Zaman, K. B. M. Q. 1985 Far-field noise of a subsonic jet under controlled excitation. J. Fluid Mech. 152, 83-111.

Zaman, K. B. M. Q. \& Hussain, A. K. M. F. 1980 Vortex pairing in a circular jet under controlled excitation. Part 1. General jet response. J. Fluid Mech. 101 (3), 449-491. 